\title{
Estimating Surface Attachment Kinetic and Growth Transition Influences on Vapor-Grown Ice Crystals
}

\author{
Gwenore F. Pokrifka, Alfred M. Moyle, Lavender Elle Hanson, And \\ JERRY Y. HARRINGTON \\ Department of Meteorology and Atmospheric Science, The Pennsylvania State University, University Park, \\ Pennsylvania
}

(Manuscript received 28 October 2019, in final form 18 February 2020)

\begin{abstract}
There are few measurements of the vapor growth of small ice crystals at temperatures below $-30^{\circ} \mathrm{C}$. Presented here are mass-growth measurements of heterogeneously and homogeneously frozen ice particles grown within an electrodynamic levitation diffusion chamber at temperatures between $-44^{\circ}$ and $-30^{\circ} \mathrm{C}$ and supersaturations $s_{i}$ between $3 \%$ and $29 \%$. These growth data are analyzed with two methods devised to estimate the deposition coefficient $\alpha$ without the direct use of $s_{i}$. Measurements of $s_{i}$ are typically uncertain, which has called past estimates of $\alpha$ into question. We find that the deposition coefficient ranges from 0.002 to unity and is scattered with temperature, as shown in prior measurements. The data collectively also show a relationship between $\alpha$ and $s_{i}$, with $\alpha$ rising (falling) with increasing $s_{i}$ for homogeneously (heterogeneously) frozen ice. Analysis of the normalized mass growth rates reveals that heterogeneously frozen crystals grow near the maximum rate at low $s_{i}$, but show increasingly inhibited (low $\alpha$ ) growth at high $s_{i}$. Additionally, 7 of the 17 homogeneously frozen crystals cannot be modeled with faceted growth theory or constant $\alpha$. These cases require the growth mode to transition from efficient to inefficient in time, leading to a large decline in $\alpha$. Such transitions may be, in part, responsible for the inconsistency in prior measurements of $\alpha$.
\end{abstract}

\section{Introduction}

Quantifying the vapor growth rate of ice crystals is challenging because it is governed by two connected processes: gas-phase diffusion and surface attachment kinetics (Markov 2003). During mass growth, water vapor molecules diffuse through the surrounding gas to reach the crystal. In the classical model of faceted growth, water molecules must then adsorb onto the crystal, though some molecules may be reflected from the surface. The fraction of molecules that adsorb onto the surface is often called a "sticking" efficiency $\beta_{s}$ and is thought to be near unity (Nelson 2001). Adsorbed molecules (ad-molecules) then migrate across the surface until they encounter an attachment site, which is provided by ledges in the crystal surface produced by dislocations in the crystal lattice or by the nucleation of two-dimensional islands on the surface. Growth ledges may also be enhanced by stacking faults, where the growth layers interchange between the cubic and hexagonal forms. Ad-molecules that reach an attachment

Corresponding author: Gwenore F. Pokrifka, gfp5025@psu.edu site can be incorporated into the crystal lattice, otherwise they will desorb from the surface. Additionally, the potential presence of quasi-liquid layers further complicates the incorporation of ad-molecules (Neshyba et al. 2016). Since most of the surface processes that govern the mass and axis growth of ice crystals have not been measured in detail, the overall influence of surface processes on growth are often represented by deposition coefficients $\alpha$ for each face. The deposition coefficient is defined as the ratio of the number of molecules incorporated to the total impinging on the crystal surface, and it can be interpreted as a growth efficiency. When $\alpha$ is near unity, the incorporation of molecules is highly efficient, and growth is limited by gas-phase diffusion (diffusion-limited growth). Small values of $\alpha(<\sim 0.001)$ correspond to inefficient molecular incorporation, such that the particle growth is limited by attachment kinetics (kinetics-limited growth). Most particles have growth rates between these two extrema (diffusion-kineticslimited growth).

Determining the influences of attachment kinetics on ice crystal growth rates has generally followed two different approaches. One approach focuses on the growth 


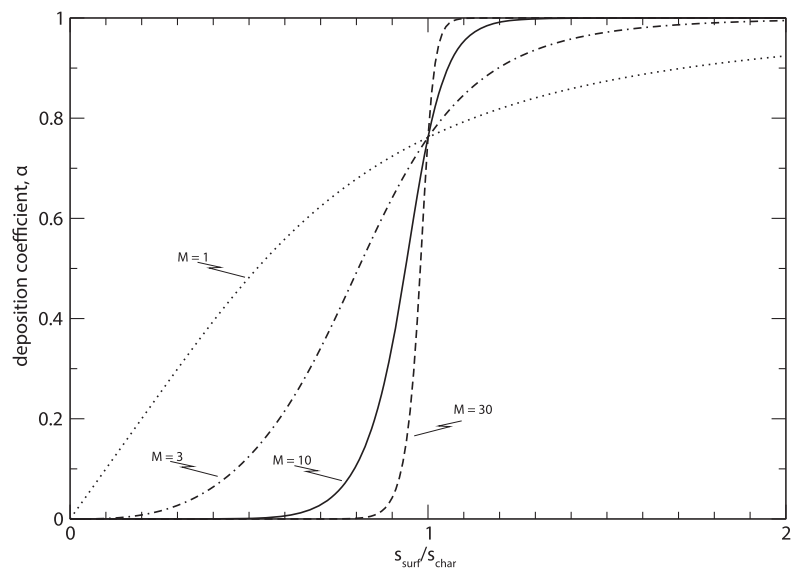

FIG. 1. Deposition coefficient as a function of the ratio of surface supersaturation to characteristic supersaturation with various growth modes $M$. A value of $M=1$ (dotted) applies to dislocation growth, $M=3$ (dot-dashed) represents stacking fault growth, and $M$ between 10 (solid) and 30 (dashed) may be used for $2 \mathrm{D}$ nucleation.

rates of crystals with fully formed facets. Faceted growth theory is well-established (see Beckmann and Lacmann 1982; Sei and Gonda 1989; Nelson and Knight 1998; Libbrecht 2003) and there is substantial evidence that facets grow primarily by two mechanisms: spiral dislocations and ledge nucleation (Nelson and Knight 1998). These mechanisms produce variable deposition coefficients that depend on the supersaturation immediately above the crystal surface (surface supersaturation $s_{\text {surf }}$ ). Spiral dislocations are permanent ledge sources producing generally efficient growth (Burton et al. 1951), with the deposition coefficient increasing steadily to unity as the $s_{\text {surf }}$ increases (Fig. 1). In contrast, ledge nucleation has a strong supersaturation dependence (Fig. 1) because $s_{\text {surf }}$ must exceed a characteristic supersaturation $s_{\text {char }}$, so that ledges will form on the surface (Nelson and Knight 1998). While classical theoretical expressions for dislocation (Burton et al. 1951) and ledge nucleation (Frank 1974) growth exist, most of the surface parameters required in those theories are unknown or cannot presently be measured. However, a convenient parameterization was formulated by Nelson and Baker (1996),

$$
\alpha=\left(\frac{s_{\text {surf }}}{s_{\text {char }}}\right)^{M} \tanh \left(\frac{s_{\text {char }}}{s_{\text {surf }}}\right)^{M},
$$

where the surface growth mechanism is described by the parameter $M$ that ranges from 1 to 30 (Fig. 1). A value of $M=1$ represents spiral dislocation growth, whereas $M \geq 10$ represents ledge nucleation. Additionally, different faces of a crystal may have different growth mechanisms, and thus different deposition coefficients. For example, a columnar crystal may have outcropping dislocations on the basal facets and ledge nucleation on the prism facets (Wood et al. 2001).

The use of faceted growth theory requires knowledge of the growth mechanism and $s_{\text {char }}$, and though both have been measured in the laboratory those measurements are sparse and generally confined to temperatures above $-40^{\circ} \mathrm{C}$. Nelson and Knight (1998) determined that, at $T>-20^{\circ} \mathrm{C}, s_{\text {char }}$ ranges between $0.15 \%$ and $2.5 \%$, and that the variation of $s_{\text {char }}$ with temperature on the basal and prism facets is consistent with the primary habits. Harrington et al. (2019) have shown that these $s_{\text {char }}$ values, in combination with ledge nucleation mediated growth, can predict the primary habits of ice at liquid saturation. Measurements of $s_{\text {char }}$ for temperatures between $-40^{\circ}$ and $-20^{\circ} \mathrm{C}$, provided by Bacon et al. (2003), Libbrecht (2003), and Libbrecht and Rickerby (2013), suggest that $s_{\text {char }}$ increases as temperature declines, with $s_{\text {char }} \sim 4 \%$ at $-20^{\circ} \mathrm{C}, s_{\text {char }} \sim 7 \%$ at $-30^{\circ} \mathrm{C}$, and $s_{\text {char }} \sim 15 \%$ at $-40^{\circ} \mathrm{C}$, which is consistent with theory. There are no precise measurements of $s_{\text {char }}$ for $T<-40^{\circ} \mathrm{C}$, though Harrington et al. (2019) have provided estimates derived from prior data.

The second approach for determining the deposition coefficient focuses on the growth of small crystals (radii $\sim 10 \mu \mathrm{m}$ ), typically those grown immediately following nucleation. Attachment kinetics have the most pronounced influence on smaller ice particles, but only as long as the deposition coefficient is constant (see Fig. 14 in Harrison et al. 2016a). Unfortunately, when this assumption is used to extract values of $\alpha$ from the mass growth of small crystals, the results are seemingly inconsistent. For example, Magee (2006) grew individual ice particles in a vertical flow levitation cell at constant temperature and pressure, but with varying supersaturation and found $\alpha$ values of about 0.006 . In contrast, Skrotzki et al. (2013) used the Aerosol Interactions and Dynamics in the Atmosphere chamber to grow populations of small crystals and determined that $\alpha$ for the population was about 0.7 . The levitation diffusion chamber studies of Harrison et al. (2016a) found $\alpha$ values ranging from about 0.005 to 0.1 . Other measurements (Choularton and Latham 1977; Haynes et al. 1992; Brown et al. 1996; Pratte et al. 2006; Earle et al. 2010; Kong et al. 2014) do not narrow the range on $\alpha$. Differences in measurement techniques and uncertainties in the ice supersaturation likely contribute to the variability in the extracted values of $\alpha$; however, it is also unlikely that even small crystals can be treated with a single, unique value of $\alpha$, an approximation that is only valid for a very narrow range of conditions (Strickland-Constable 1968, 160-161; 
Nelson 2005). It is more likely that the physical properties of the ice particle surfaces varied among experiments, and during the growth of each individual crystal.

Ice crystals can transition from one growth mechanism to another over time. For example, an ice particle's dominant growth mechanism depends upon the ambient ice supersaturation $s_{i}$ (hereafter "supersaturation") and the particle size. Immediately after a crystal forms by nucleation it is likely that numerous dislocations exist in the crystal lattice, and these dominate the growth as long as $s_{i}$ remains low (Burton et al. 1951; Nelson 2001; Harrington et al. 2019). However, ledge nucleation must take over as the dominant growth mechanism at higher supersaturations and as crystal facets become larger, otherwise it would not be possible to produce thin plates and columns (Frank 1982; Nelson and Knight 1998; Harrington et al. 2019).

Growth transitions also occur immediately following ice nucleation. The measurements of Gonda and Yamazaki (1978) show such a process as frozen droplets transition first to droxtals and finally to hexagonal prisms. The droxtal morphology consists of the lowindex basal and prism facets of hexagonal ice, which grow slowly, and higher-index pyramidal facets that grow rapidly (examples in Figs. 1 and 2 from Nelson and Swanson 2019). Gonda and Yamazaki (1984) showed that the pyramidal regions grew rapidly until they disappeared, leaving only the slowly growing basal and prism facets, and a hexagonal single crystal. The transition took only $2-4 \mathrm{~min}$ to be completed at $-15^{\circ} \mathrm{C}$ and low supersaturation $(2 \%)$.

Similarly, droplets frozen at temperatures below $-20^{\circ} \mathrm{C}$ are often polycrystalline and develop facets over time (cf. Bacon et al. 2003). The interface between crystalographically dissimilar facets in polycrystalline ice (grain boundary), is a known source of dislocations (Furukawa and Kobayashi 1978) and can cause increased growth rates (Pedersen et al. 2011). Therefore, when a polycrystal is small, the growth may be dominated by dislocations and be relatively efficient. However, as facets grow away from the grain boundaries, such as the arms of an emerging bullet rosette, the central portions of the crystal may be effectively starved of water vapor (Westbrook et al. 2008). Growth would then be dominated by the exposed facets. This transition apparently occurs more slowly than that of the single crystals discussed above; the results of Bacon et al. (2003) indicate that it may take tens of minutes before rosette arms fully emerge. The key point is that we should expect to find transitions in crystal growth mechanisms as facets emerge following ice nucleation in laboratory experiments that focus on newly formed ice crystals. We will refer to such variations in the dominant growth mechanism as "kinetics transitions."

In this article we provide estimates of the growth rates for heterogeneously and homogeneously nucleated crystals grown from the vapor in the button electrode levitation (BEL) diffusion chamber (Harrison et al. 2016a). We develop new analysis methods that allow for the determination of $\alpha$ without the uncertainty inherent in the measured ambient supersaturation, which potentially biased prior estimates of $\alpha$. The results indicate that the variability in the deposition coefficient may be due to variations in the growth mechanism of individual particles. Moreover, the analysis provides evidence that the growth rate depends on the nucleation mechanism, and that homogeneously frozen ice exhibits evidence of kinetics transitions. In the following sections, we briefly describe our experimental procedure, review our growth model and analysis methods, and discuss the main results.

\section{Diffusion chamber and ice growth experiments}

The BEL chamber is described in detail in Harrison et al. (2016a), and therefore only a brief description of the chamber and its operating principles is provided here. The BEL chamber is a thermal gradient diffusion chamber, making it advantageous for growth experiments because it combines the stable thermal and supersaturated environment of a classic diffusion chamber with electrodynamic particle levitation. The growth chamber is a cylinder defined by the adjacent surfaces of two parallel copper plates, separated by $1.27 \mathrm{~cm}$, and a Plexiglas ring, with an inner diameter of $10.2 \mathrm{~cm}$, that composes the outer wall. The cylinder aspect ratio of 8:1 is large enough to avoid the possibility of wall effects (Elliott 1971). The temperature of each plate is controlled independently, and held constant, by circulating cryogen through Plexiglas housings on each plate. The BEL chamber operates at ambient atmospheric pressure $(\sim 970 \mathrm{hPa})$, which we use in our data analysis.

A charged water droplet, composed of high-pressure liquid chromatography (HPLC) water from J. T. Baker, is launched into the chamber. The bottom copper plate is given a direct current voltage that produces a vertically positive electric field such that the resulting electrostatic force on the particle counteracts gravity. This voltage is adjustable and is used to track changes to the levitating particle's mass. The top plate is a grounded electrode. Four button electrodes on the top plate receive alternating current voltages and act as a quadrupole, producing a saddle point that centers and stabilizes the levitating particle. Furthermore, the saddle point has a net zero vertical force, such that it does not influence the 
voltage required to levitate the particle. Additionally, the amount of charge on the particle is an order of magnitude smaller than what is required to produce electrically enhanced growth, and such growth is therefore unlikely (Davis 2010). A 5-mW, 632.8-nm heliumneon laser is used to illuminate the particle, providing both particle position and scattering diffraction patterns. The particle initial radius is determined (to typically within $1 \mu \mathrm{m}$ ) by matching the measured diffraction patterns with Mie theory while the particle is still liquid, as in our prior work (Xue et al. 2005; Harrison et al. 2016a). A Python program developed by our group maintains the particle vertical location by adjusting the bottom plate voltage. We use the recorded bottom-plate voltage and the initial particle size to determine the particle mass, since the ratio of the voltage to its initial value is equivalent to the mass, $m$, normalized to the initial mass $m_{0}$. We use the mass ratio, $m_{r} \equiv m / m_{0}$, in our analysis because it is a directly measured quantity with little uncertainty. The mass ratio has an estimated maximum relative error of $5 \%$, which we use in our analysis below.

Supersaturation in the chamber is produced by diffusive transport of water vapor from the upper, warmer plate to the lower, colder plate. Filter paper soaked in HPLC water is attached to both plates to serve as the vapor source, though there are holes in the filter paper on the top plate for the electrodes and the launcher opening. An experiment at low supersaturation suggests that these holes have little impact on the supersaturation at the particle location (see appendix). Moreover, the experiment provides evidence that the supersaturation is near the value determined from standard, flat-plate diffusion chamber theory (e.g., Elliott 1971), which is used here. Supersaturation uncertainty is from the plate temperatures $\left( \pm 0.2^{\circ} \mathrm{C}\right)$ and chamber depth $( \pm 0.1 \mathrm{~mm})$ typically resulting in a relative uncertainty of about $10 \%$.

\section{Analysis methods}

\section{a. Vapor growth model}

Interpretation of laboratory data requires a flexible model of vapor growth that includes the influences of attachment kinetics. While hexagonal and cylindrical models of crystal growth exist (Nelson and Baker 1996; Wood et al. 2001), a simplified approach is better suited here, given the unknown geometry of the growing crystals. We use the Diffusion Surface Kinetics Ice Crystal Evolution (DiSKICE) model (Zhang and Harrington 2014), which approximates ice as a spheroid with two dimensions $a$ and $c$. The DiSKICE model is valid for faceted growth, it compares well to hexagonal model solutions (Zhang and Harrington 2014), and has been successful in the interpretation of laboratory growth data (Harrison et al. 2016a; Harrington et al. 2019). DiSKICE uses capacitance theory to model the far-field gas-phase diffusion to the particle,

$$
\frac{d m}{d t}=4 \pi C(c, a) s_{i} \underbrace{\rho_{\mathrm{eq}}\left[\frac{1}{D_{v}}+\frac{\rho_{\mathrm{eq}} l_{s}}{K_{T} T}\left(\frac{l_{s}}{R_{v} T}-1\right)\right]^{-1}}_{\equiv D},
$$

where $C(c, a)$ is the capacitance that depends on the $a$ and $c$ semiaxis lengths, $D_{v}$ is the vapor diffusivity in air, $K_{T}$ is the thermal conductivity of air, $R_{v}$ is the vapor gas constant, $l_{s}$ is the enthalpy of sublimation, $\rho_{\mathrm{eq}}$ is the ice equilibrium vapor density, and $D$ is a growth factor that combines the vapor and thermal diffusivity (hereafter "vapor-thermal diffusivity") following the form in Lamb and Verlinde (2011). In DiSKICE, the vapor diffusivity $D_{v}$ is replaced with a kinetically modified vapor diffusivity $D_{\text {kin }}$ that includes the deposition coefficients for the two primary axes ( $a$ and $c$ ) of the crystal $\left(\alpha_{a}\right.$ and $\left.\alpha_{c}\right)$,

$$
D_{\mathrm{kin}}=\frac{2}{3} \frac{D_{v}}{\left(\frac{4 D_{v} C}{\alpha_{c} \bar{v}_{v} a c}+\frac{C}{C_{\Delta}}\right)}+\frac{1}{3} \frac{D_{v}}{\left(\frac{4 D_{v} C}{\alpha_{a} \bar{v}_{v} a^{2}}+\frac{C}{C_{\Delta}}\right)} .
$$

Here, $\bar{v}_{v}$ is the mean speed of a vapor molecule and $C_{\Delta}$ is the capacitance evaluated a distance $\Delta$ (the vapor mean free path) away from the crystal surface. Once $D_{\text {kin }}$ replaces $D_{v}$ in Eq. (2), the vapor-thermal diffusivity $D$ becomes an "effective" diffusivity $\left[D_{\text {eff }} \equiv D\left(\alpha, K_{T}, C\right)\right]$ that depends on attachment kinetics. Equation (3) combines vapor diffusion in air with attachment kinetics that depend on size and supersaturation through $\alpha$ [Eq. (1)]. Attachment kinetics therefore reduce the mass growth of a crystal since $D_{\text {kin }}$ is generally less than $D_{v}$; however, as $\alpha$ approaches unity DiSKICE approaches the capacitance solution, or diffusion-limited growth.

The particles grown in our diffusion chamber are relatively small (typical maximum radius of $30-40 \mu \mathrm{m}$ ), and are likely isometric. Therefore our analysis will assume spherical growth, so that $a=c \equiv r$ and $\alpha_{a}=\alpha_{c} \equiv \alpha$. However, we examine the theoretical limits of this assumption through calculations of nonisometric growth, which is treated in DiSKICE with a theory to distribute mass along the crystal axes based on Nelson and Baker (1996),

$$
\frac{d c}{d a}=\frac{\alpha_{c}}{\alpha_{a}} \equiv \Gamma
$$

This equation is valid for faceted growth and assumes that ledge growth begins at crystal edges (Nelson and Baker 1996). 
Its use with Eqs. (1) and (2) produces relatively accurate evolution of single crystalline ice (Harrington et al. 2019).

\section{b. Scaled growth-rate analysis}

Prior studies of the growth of small ice crystals have often directly analyzed the time series of the particle mass, and mass derivative (e.g., Magee et al. 2006). This approach, however, requires knowledge of the supersaturation, which is challenging to measure accurately in most laboratory devices. Uncertainty in the supersaturation has led to questions regarding the accuracy of deposition coefficient estimates made from measured growth rates (Magee et al. 2006; Skrotzki et al. 2013; Harrison et al. 2016a). It is therefore advantageous to develop an analysis procedure that does not directly depend on $s_{i}$. The analysis method developed below uses the time-averaged growth rate, which can be determined directly from the data, as a proxy for the supersaturation. The ice particle growth rate is scaled by the time-averaged value effectively removing the direct $s_{i}$ dependence from the analysis. This analysis, or a modified version, may be useful for any single ice particle growth experiment where $s_{i}$ is constant.

We begin by assuming that our small particles are spherical $(C=r)$ and average over the entire growth time series [Eq. (2)] such that

$$
\overline{\dot{m}}=4 \pi \overline{r D_{\text {eff }}} s_{i} .
$$

Since $s_{i}$ is independent of time, we can use Eq. (5) to replace $s_{i}$ in Eq. (2), which gives the mass growth rate in terms of its time average,

$$
\dot{m}=\frac{r D_{\text {eff }}}{\overline{r D_{\text {eff }}}} \overline{\dot{m}}=\frac{r D_{\text {eff }}}{\bar{r} \overline{D_{R}}} \overline{\dot{m}}, \quad \text { where } \quad \overline{D_{R}}=\frac{\sum r D_{\text {eff }}}{\sum r}
$$

is the radius-weighted average effective diffusivity. We next normalize Eq. (6) by the mean mass growth rate $\overline{\dot{m}}$, and rewrite it in terms of the mass ratio $m_{r}$ :

$$
\frac{\dot{m}_{r}}{\dot{\bar{m}}_{r}}=\frac{m_{r}^{1 / 3} D_{\text {eff }}}{\overline{m_{r}^{1 / 3}} \overline{D_{R}}}
$$

which applies to spherical, diffusion-kinetics growth.

An advantage of Eq. (7) is that the diffusion and kinetics limits can be determined directly from the data. In diffusion-limited growth, where $\alpha \rightarrow 1, D_{\text {kin }}$ approaches $D_{v}, D_{\text {eff }}$ is constant, and the capacitance model [Eq. (2)] is recovered, leading to

$$
\frac{\dot{m}_{r}}{\overline{\dot{m}}_{r}}=\frac{r}{\bar{r}}=\frac{m_{r}^{1 / 3}}{m_{r}^{1 / 3}} .
$$

Conversely, when growth is kinetics-limited $(\alpha \rightarrow 0)$ gas-phase diffusion no longer limits the growth rates. In this case, $D_{\text {eff }}$ approaches $\alpha r$ [Harrington et al. 2009, their Eq. (15)] and the mass growth rate becomes proportional to $r^{2}$ :

$$
\frac{\dot{m}_{r}}{\dot{m}_{r}}=\frac{r^{2}}{\overline{r^{2}}}=\frac{m_{r}^{2 / 3}}{m_{r}^{2 / 3}}
$$

Instead of using Eqs. (7)-(9) in our analysis, we have found it more convenient to use a scaled mass growth rate $G_{s}$, which is defined using the kinetics limit as a basis,

$$
G_{s} \equiv \frac{\dot{m}_{r}}{\dot{m}_{r}} \overline{m_{r}^{2 / 3}}
$$

Finally, we take the natural logarithm of $G_{s}$, and following the same procedure for Eqs. (7) and (8) gives

\author{
(diffusion-kinetics-limited growth) \\ (diffusion-limited growth) \\ (kinetics-limited growth).
}

All of the terms in Eq. (11) can be determined directly from the data, with the exception of the effective diffusivity terms. The effective diffusivity depends weakly on temperature and strongly on the deposition coefficient and size. The DiSKICE model can be used to determine $D_{\text {eff }} / \overline{D_{R}}$ and, therefore, $\alpha$ through best fits to the scaled mass growth rate. This procedure is advantageous because it does not 

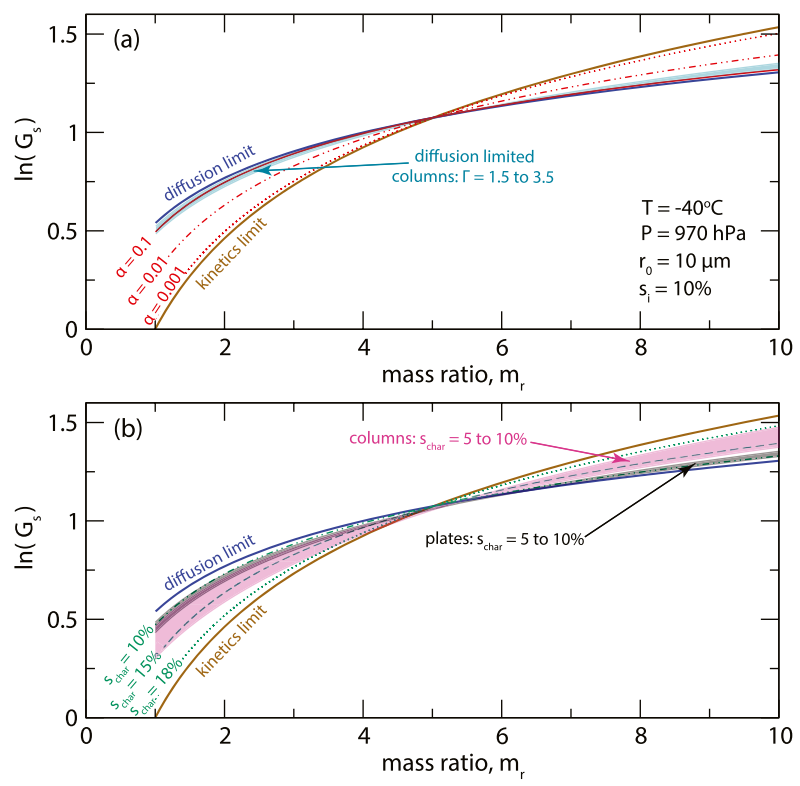

FIG. 2. DiSKICE model output of the natural logarithm of the scaled mass growth rate as a function of the mass ratio with either constant (a) $\alpha$ or (b) $s_{\text {char }}$. Results for both spheres (curves) and nonisometric particles (shading) are plotted. Solid blue curves represent the diffusion limit to growth $(\alpha=1)$, and brown curves are the kinetics limit $(\alpha \rightarrow 0)$. In (a) the diffusion limit of columnar growth with deposition coefficient ratio $\Gamma$ between 1.5 and 3.5 is in blue shading. In (b) nonisometric growth with the characteristic supersaturation on the major axis ranging between $5 \%$ and $10 \%$ is shaded in pink for columns and gray for plates.

require the supersaturation, which is not directly measured, and it uses the mass ratio, which we directly measure with low uncertainty.

Theoretical calculations of $G_{s}$ using DiSKICE illustrate the advantages and limitations of this approach. Figure 2 shows an example calculation for a spherical ice particle growing from an initial radius of $10 \mu \mathrm{m}$ at a temperature of $-40^{\circ} \mathrm{C}$ and $s_{i}=10 \%$, conditions similar to our experiments. Note that $\ln \left(G_{s}\right)$ curves for the diffusion and kinetics limits intersect once, and produce a bounding region for diffusion-kinetics growth. Ice particles undergoing diffusion-kinetics-limited growth were simulated either with a constant $\alpha$ (Fig. 2a) or using ledge nucleation $(M=10)$ with a given $s_{\text {char }}$ (Fig. 2b). In either case, when $\alpha$ is large $(\alpha>0.1)$ or $s_{\text {char }}$ is small (which produces larger $\alpha$ ), the scaled growth rate is near the diffusion limit. Particles growing with lower $\alpha$ or higher $s_{\text {char }}$ follow curves that are between the diffusion and kinetics limit, but the curves intersect at the same location.

The scaled growth rate analysis is limited in that it is strictly valid for spherical (isometric) particles. Our measured crystals remain small, but some nonisometric growth may occur. It would then be useful to know

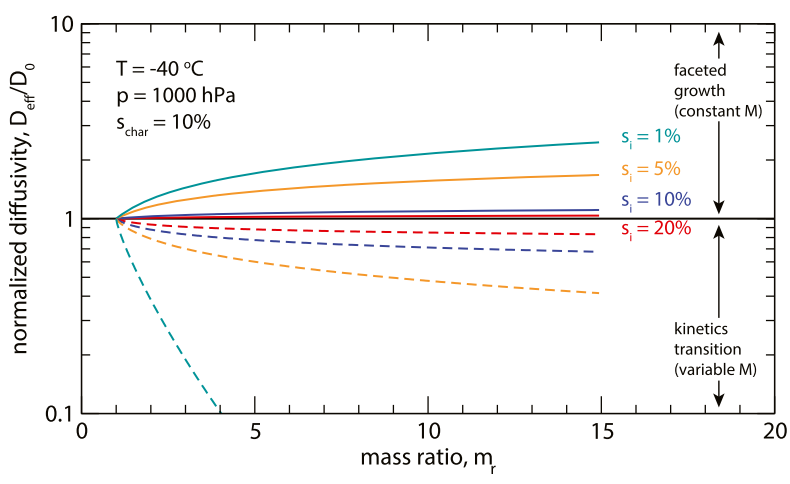

FIG. 3. DiSKICE model output of the normalized diffusivity as a function of the mass ratio at various supersaturations $(1 \%, 5 \%$, $10 \%$, and $20 \%$ ). The solid curves correspond to faceted growth, where a growth mechanism parameter $M=10$ was used. The dashed curves varied $M$ according to Eq. (15) to simulate a kinetics transition.

whether nonisometric growth at the diffusion limit (high $\alpha$ ) could be falsely identified as diffusion-kineticslimited growth (low $\alpha$ ), in particular. DiSKICE simulations indicate that false identification is unlikely since diffusion-limited columns and plates (aspect ratios between 0.1 and 10) have scaled mass growth rates that do not deviate far from the diffusion limit calculated for spherical crystals (Fig. 2a). Additionally, nonisometric crystals growing by ledge nucleation $\left(s_{\text {char }}\right.$ between $5 \%$ and $10 \%$ ), with low $\alpha$, have scaled growth rates that fall within the kinetics and diffusion limits determined for spherical growth (Fig. 2b). However, it is possible for moderate kinetics influence on nonisometric growth to be misidentified as diffusion limited (i.e., $s_{\text {char }}<10 \%$ ). That is, the scaled growth rate analysis for nonisometric particles can produce false-positive results of diffusionlimited growth, even though the crystals grew with kinetics limitations. Together, these results indicate that deviations from the diffusion limit provide an estimate of the overall kinetics limitations of growing particles, even if the particles are nonspherical. However, this method alone does not conclusively identify diffusionlimited growth.

\section{c. Power-law analysis}

A second analysis method that is independent of the supersaturation originates from expressing the mass growth rate of an ice crystal in a power-law form with respect to size or mass. Often, $d m / d t \propto m^{P / 3}$, where $P=1$ for diffusion-limited growth [Eq. (8)] and $P=2$ for kinetics-limited growth [Eq. (9)] (see also Swanson et al. 1999). This form is possible because the effective diffusivity, $D_{\text {eff }}$ in Eq. (2), has a size-dependent power law as shown by the solid curves in Fig. 3. We can therefore write the effective diffusivity as 


$$
D_{\text {eff }}=D_{0} m_{r}^{n / 3},
$$

where $D_{0}$ is the effective diffusivity at the initial particle size $\left(m_{r}=1\right)$. For faceted growth, the exponent $n$ takes a value between 0 (diffusion limited) and 1 (kinetics limited). Rewriting Eq. (2) in terms of the mass ratio, substituting in Eq. (12) for $D$, and normalizing by the initial growth rate $\dot{m}_{0}$, shows that for diffusion-kineticslimited growth

$$
\frac{\dot{m}}{\dot{m}_{0}}=m_{r}^{(1+n) / 3}
$$

We isolate the power exponent, $1+n$, by taking the natural logarithm of both sides of Eq. (13) and rearranging such that

$$
3 \frac{\ln \left[\frac{\dot{m}}{\dot{m}_{0}}\right]}{\ln \left[m_{r}\right]}=1+n \equiv P .
$$

The power exponent $P$ is independent of the supersaturation and is only weakly dependent on the initial particle radius. Moreover, note that the left-hand side of Eq. (14) can be computed directly from the mass data without the use of a growth model. In our analysis, we use $\dot{m}_{0}$ with a short time average to remove highfrequency noise (see section $4 \mathrm{a}$ ).

Theoretical calculations with the DiSKICE model reveal the advantages and limits of the power-law approach (Fig. 4). Like the scaling analysis, $P$ values for isometric growth are confined between the diffusion and kinetics limits, with larger deposition coefficients and smaller characteristic supersaturations resulting in values of $P$ nearer the diffusion limit. Diffusion-limited nonisometric growth produces values of $P$ that do not deviate far from the diffusion limit for spherical growth, indicating that this method, like the scaled growth rate, will not falsely identify diffusion-limited, nonspherical growth as kinetically limited. These calculations also suggest that values of $P \geqslant 1.1$ indicate growth is limited, in part, by attachment kinetics, regardless of particle shape. The power-law analysis provides a robust estimate of attachment kinetics influence on growth, like the scaled growth rate analysis. Unlike that method, however, the shape of the curve differs for isometric and nonisometric growth, which can produce $P<1$ during early stages of nonisometric growth.

\section{Results}

Mass ratio time series taken from ice crystals grown in the BEL chamber are analyzed in this section. Ice crystals were nucleated both homogeneously (Pokrifka
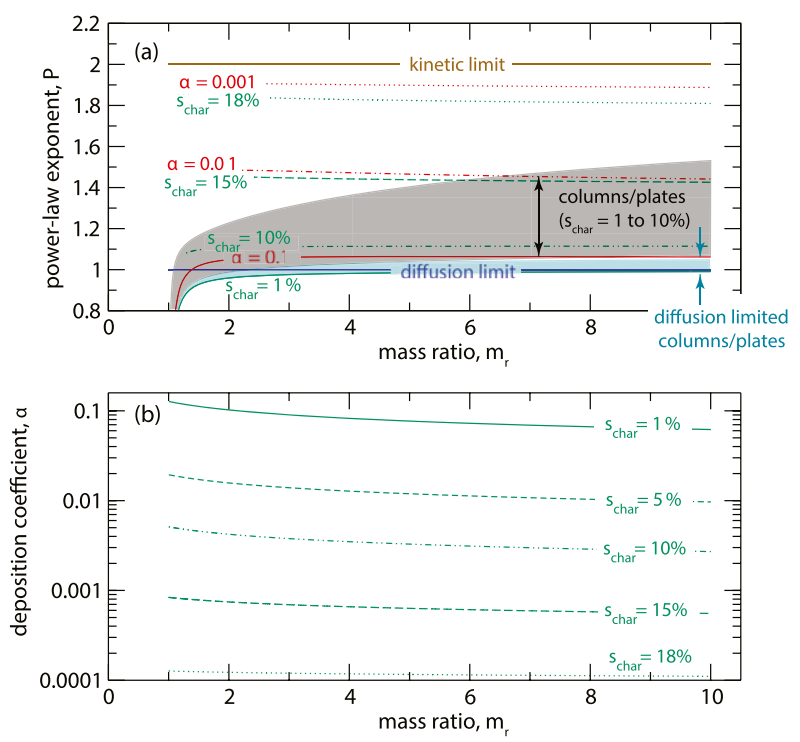

FIG. 4. (a) DiSKICE model simulations of the power-law analysis with constant $\alpha$ (red) or ledge nucleation $(M=10)$ with a given $s_{\text {char }}$ (green). (b) The deposition coefficients for each characteristic supersaturation are also plotted. Results for nonisometric particles are plotted in shaded regions for the diffusion limit (blue) and variable $\alpha$ (gray). The remaining conditions for these simulations are as in Fig. 2.

et al. 2018) and heterogeneously using the bionucleant Snomax (Harrison et al. 2016b) in the experiments. The time series of the ice crystals nucleated heterogeneously were presented in a prior work (Harrison et al. 2016a) and will be used in the analysis here. In Table 1, we give the temperatures and supersaturations under which the particles grew, and their initial radii (generally $r_{0}<15 \mu \mathrm{m}$ ). We do not include the final particle sizes, but they rarely grow beyond a radius of $60 \mu \mathrm{m}$. Figure 5 shows a representative sample of the data, including two experiments that produced very long periods of growth, though the particles usually grew for less than $1500 \mathrm{~s}$. The data were originally recorded at $10-30 \mathrm{~Hz}$, thus we plot the data at a much lower frequency to avoid cluttering the figure, but do include enough points such that the trend is obvious. Though the growth time series for heterogeneously and homogeneously nucleated ice appear to be similar [cf. Fig. 5 herein to Fig. 4 from Harrison et al. (2016a)], analysis of the growth rates reveals important differences.

\section{a. Refining raw data}

Since our analysis methods require the mass ratio derivatives, the particle time series must be smoothed to remove noise and low-frequency features caused by the software controlling particle levitation. We use a lowpass filter with a sliding window that is operated forward 
TABLE 1. Experimental conditions, temperature $T$ and supersaturation $s_{i}$, and initial particle radii $r_{0}$. The data from heterogeneous freezing experiments are in Harrison et al. (2016b).

\begin{tabular}{|c|c|c|c|c|c|}
\hline \multicolumn{3}{|c|}{ Homogeneous freezing experiments } & \multicolumn{3}{|c|}{ Heterogeneous freezing experiments } \\
\hline$T\left({ }^{\circ} \mathrm{C}\right)$ & $s_{i}(\%)$ & $r_{0}(\mu \mathrm{m})$ & $T\left({ }^{\circ} \mathrm{C}\right)$ & $s_{i}(\%)$ & $r_{0}(\mu \mathrm{m})$ \\
\hline$-43.5 \pm 0.1$ & $17.2 \pm 1.5$ & $10.55 \pm 0.16$ & $-35.7 \pm 0.1$ & $28.6 \pm 2.1$ & $8.9 \pm 0.7$ \\
\hline$-43.5 \pm 0.1$ & $17.4 \pm 1.5$ & $9.92 \pm 0.38$ & $-35.7 \pm 0.1$ & $28.6 \pm 2.1$ & $8.2 \pm 0.5$ \\
\hline$-43.4 \pm 0.1$ & $12.9 \pm 1.3$ & $21.55 \pm 0.35$ & $-35.7 \pm 0.1$ & $28.6 \pm 2.1$ & $9.2 \pm 0.5$ \\
\hline$-43.4 \pm 0.1$ & $17.1 \pm 1.4$ & $8.65 \pm 0.39$ & $-35.7 \pm 0.1$ & $28.6 \pm 2.1$ & $10.3 \pm 0.5$ \\
\hline$-42.4 \pm 0.1$ & $17.7 \pm 1.5$ & $10.82 \pm 0.42$ & $-33.8 \pm 0.1$ & $17.0 \pm 1.1$ & $9.86 \pm 0.25$ \\
\hline$-42.2 \pm 0.1$ & $18.1 \pm 1.5$ & $15.05 \pm 0.43$ & $-33.8 \pm 0.1$ & $17.0 \pm 1.1$ & $8.9 \pm 0.2$ \\
\hline$-42.1 \pm 0.1$ & $18.8 \pm 1.5$ & $10.60 \pm 0.20$ & $-33.0 \pm 0.1$ & $10.0 \pm 1.1$ & $8.7 \pm 0.3$ \\
\hline$-40.2 \pm 0.1$ & $13.5 \pm 1.3$ & $9.25 \pm 0.32$ & $-33.0 \pm 0.1$ & $10.0 \pm 1.1$ & $5.8 \pm 0.4$ \\
\hline$-38.4 \pm 0.1$ & $3.7 \pm 0.4$ & $26.43 \pm 1.4$ & $-33.0 \pm 0.1$ & $10.0 \pm 1.1$ & $7.6 \pm 0.3$ \\
\hline$-37.8 \pm 0.1$ & $7.5 \pm 0.7$ & $11.65 \pm 1.2$ & $-32.6 \pm 0.1$ & $11.0 \pm 0.9$ & $10.44 \pm 0.15$ \\
\hline$-37.4 \pm 0.1$ & $11.0 \pm 1.1$ & $7.59 \pm 0.85$ & $-32.6 \pm 0.1$ & $11.0 \pm 0.9$ & $11.7 \pm 0.2$ \\
\hline$-37.4 \pm 0.1$ & $11.0 \pm 1.1$ & $9.86 \pm 0.88$ & $-32.0 \pm 0.1$ & $7.5 \pm 0.7$ & $9.6 \pm 0.3$ \\
\hline$-37.4 \pm 0.1$ & $7.5 \pm 0.7$ & $11.34 \pm 1.0$ & $-31.7 \pm 0.1$ & $7.5 \pm 0.7$ & $6.3 \pm 0.4$ \\
\hline$-37.0 \pm 0.1$ & $14.0 \pm 1.4$ & $10.0 \pm 0.70$ & $-31.7 \pm 0.1$ & $7.5 \pm 0.7$ & $12.0 \pm 0.1$ \\
\hline$-36.7 \pm 0.1$ & $14.1 \pm 1.4$ & $7.84 \pm 0.74$ & $-31.5 \pm 0.1$ & $5.0 \pm 0.6$ & $10.69 \pm 0.17$ \\
\hline$-36.6 \pm 0.1$ & $18.7 \pm 1.5$ & $13.2 \pm 1.5$ & $-30.9 \pm 0.1$ & $4.0 \pm 0.5$ & $8.2 \pm 0.4$ \\
\hline$-36.6 \pm 0.1$ & $14.2 \pm 1.4$ & $9.45 \pm 0.76$ & $-30.9 \pm 0.1$ & $4.0 \pm 0.5$ & $7.7 \pm 0.3$ \\
\hline$-36.3 \pm 0.1$ & $4.6 \pm 0.4$ & $16.83 \pm 0.76$ & & & \\
\hline
\end{tabular}

and backward. Filtering both forward and backward avoids the phase shift that occurs from forward-only filtering. A different window width is used in each case, such that noise is sufficiently removed and and the derivatives can be computed. However, a window's width of data are always lost at the beginning and end of the time series, and some low-frequency oscillations are only partially removed by the filter. Because lowerfrequency features are challenging to remove, we also analyze the data by first fitting them with cubic polynomials following Magee (2006). Doing so effectively removes the low-frequency features but retains the overall growth trend. We find that both of these methods of data smoothing produce time series and derivatives in close agreement with the raw data (Fig. 6). Note that the derivatives of the low-pass-filtered and cubic-fit data both follow the same trend as the smoothed growth rate determined from the raw data, and all three fall within the uncertainty range determined from the uncertainty on $m_{r}$. The smoothing process shown in Fig. 6 is similarly accurate for all of the other datasets. Because low-frequency features are removed by the cubic fits to the data, they are used in the analysis below, unless otherwise stated.

\section{b. Scaled growth rate results}

Applying the scaled growth analysis from section $3 \mathrm{~b}$ to the heterogeneously frozen drops indicates that each individual time series can be modeled by faceted growth theory [Eqs. (1)-(3)]. For example, the crystal in Fig. 7 has a scaled growth rate that lies within the diffusion- and kinetics-limit boundaries (Fig. 7a), and has a curve shape similar to faceted growth theory (Fig. 2). As in Harrison et al. (2016a), we fit the data with DiSKICE assuming a constant $\alpha$. This is justifiable if the particles grow by a single growth mechanism $[M=$ constant in Eq. (1)] and they remain small $(r \sim 10-30 \mu \mathrm{m})$. DiSKICE model fits to both the low-pass-filtered data and the cubicfit data accurately simulate the particles' scaled and actual growth rates (Fig. 7b). The case shown in Fig. 7 is clearly limited by attachment kinetics, and the model fits to the data indicate that $\alpha=0.008 \pm 0.002$, with the uncertainty determined by using the uncertainty in the mass ratio growth rates. This $\alpha$ is similar to the $\alpha=0.009 \pm 0.003$ determined by Harrison et al. (2016a) using fits to the mass ratio time series. All of the heterogeneously frozen droplets have scaled growth rates similar to that shown in Fig. 7, with varying degrees of kinetic limitation.

Originally, Harrison et al. (2016a) determined that all of their particles grew with deposition coefficients less than 0.1 , but the scaled growth rate analysis indicates that the low $s_{i}$ cases grew with somewhat higher $\alpha$, sometimes near unity. Since the scaled growth rate analysis uses the measured mean-mass growth rate instead of the unmeasured supersaturation used by Harrison et al. (2016a), these new results are arguably more reliable. Consequently, these results suggest that the supersaturation determined from the twodimensional chamber model of Harrison et al. (2016a) may be too uncertain when $s_{i}$ is low. The chamber model is most sensitive to the uncertainty in the ice coverage on 

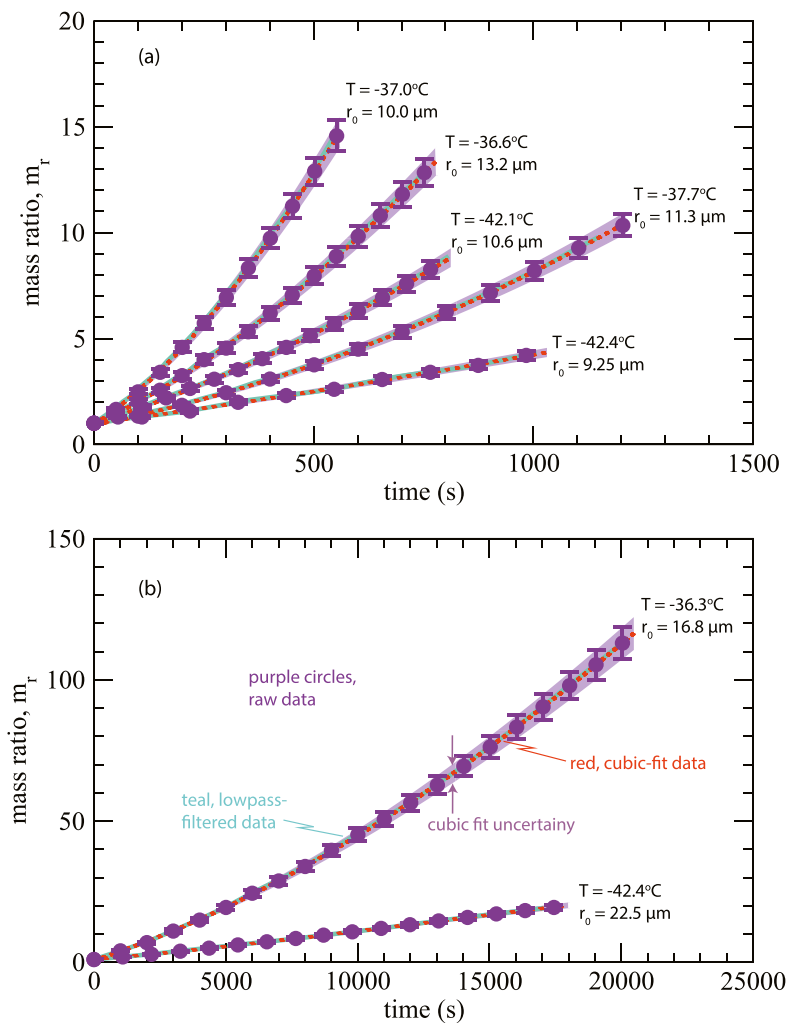

FIG. 5. Representative mass ratio growth time series for homogeneously frozen ice particles. Purple points are raw data with an uncertainty of $5 \%$. The data are smoothed with a low-pass filter (solid teal) and a cubic fit (dashed red); the latter uncertainty range of $5 \%$ (purple shading) is also shown.

the top plate in low $s_{i}$ conditions, understandably leading to the largest errors occurring at the lowest $s_{i}$. The low $s_{i}$ experiments from Harrison et al. (2016a) are, therefore, modified the most by the scaled analysis, though the general variability of $\alpha$ with temperature (green points in Fig. 8) is similar to that shown in Fig. 13 of Harrison et al. (2016a). The scaled growth analysis shows that the heterogeneously frozen particles demonstrate both growth that is diffusion limited or diffusion-kinetics limited, as $\alpha$ ranges between 0.008 and unity.

Ten of the 17 experiments with homogeneously frozen droplets grow similarly to the heterogeneous freezing cases. Some data show growth at the diffusion limit (Fig. 9), and others demonstrate the influence of attachment kinetics (Fig. 10). In all of these cases, there is good agreement between the DiSKICE model fits to the scaled growth rate and mass ratio growth rate, as determined from both the low-pass-filtered and the cubic-fit data. These results indicate that the classical model of faceted growth fits the general growth features of the data.

There are, however, 7 of the 17 cases where the homogeneously frozen ice growth data cannot be modeled
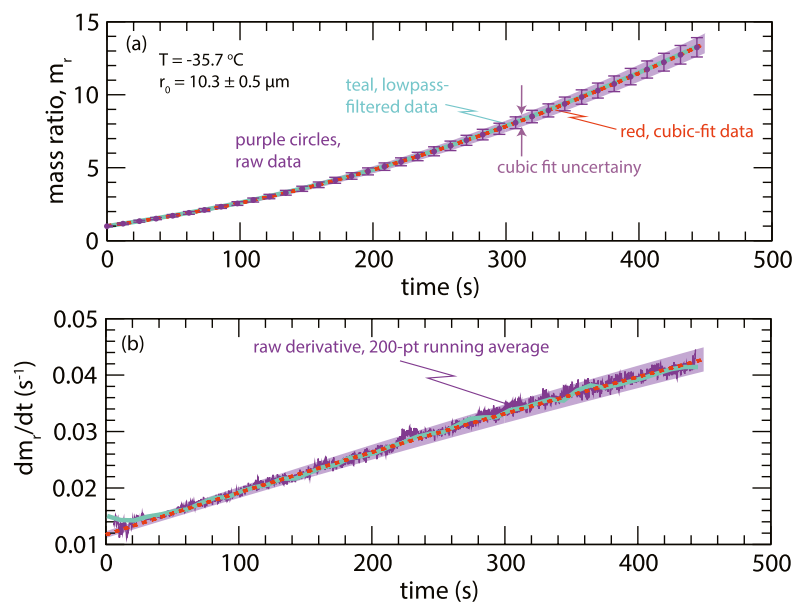

FIG. 6. (a) Mass ratio growth time series of a heterogeneously frozen ice particle. Points and curves follow the same scheme as in Fig. 5. (b) Time derivative of the data in (a) where the points are replaced by a 200-point running average (solid purple) and the uncertainty range (purple shading). Derivatives of the low-passfiltered and cubic-fit data are given as the solid teal and dashed red curves, respectively.

using current theory. These cases have scaled growth rates that reside outside of the region between the diffusion and kinetics limits (Fig. 11a), and they have nearly constant mass growth rates (Fig. 11b). It is worth noting that similar growth rates have been observed by Gonda and Yamazaki (1984, their Fig. 3) and Nelson and Swanson (2019, their Fig. 5) for crystals with emerging facets, where the growth rates initially rise, but asymptote in time. If our crystals grew similarly, it is then not surprising that fitting the growth rates assuming faceted growth using DiSKICE initially underestimates, then overestimates, the growth rates (Fig. 11, black curve). Recall that the mass growth rate is proportional to the ice particle size and the effective diffusivity [Eq. (2)], both of which increase in time for faceted growth. Thus, a constant mass growth rate in a static environment is only achievable by decreasing the effective diffusivity with size, which only occurs if the deposition coefficient falls as the particle size increases. This is consistent with what can occur during a kinetics transition, when fast growing regions disappear, or become shadowed, leaving only slow growing regions, and causing a substantial decline in the deposition coefficient.

Other processes that can produce unusual growth rates include changing aspect ratios, the loss or gain of charge on the particle, and gas contamination. Changes in aspect ratio generally lead to increases in growth rate with size, not decreases. Charge loss or gain has never been detected in prior studies with the BEL chamber. For instance, equilibrated solution droplets were 

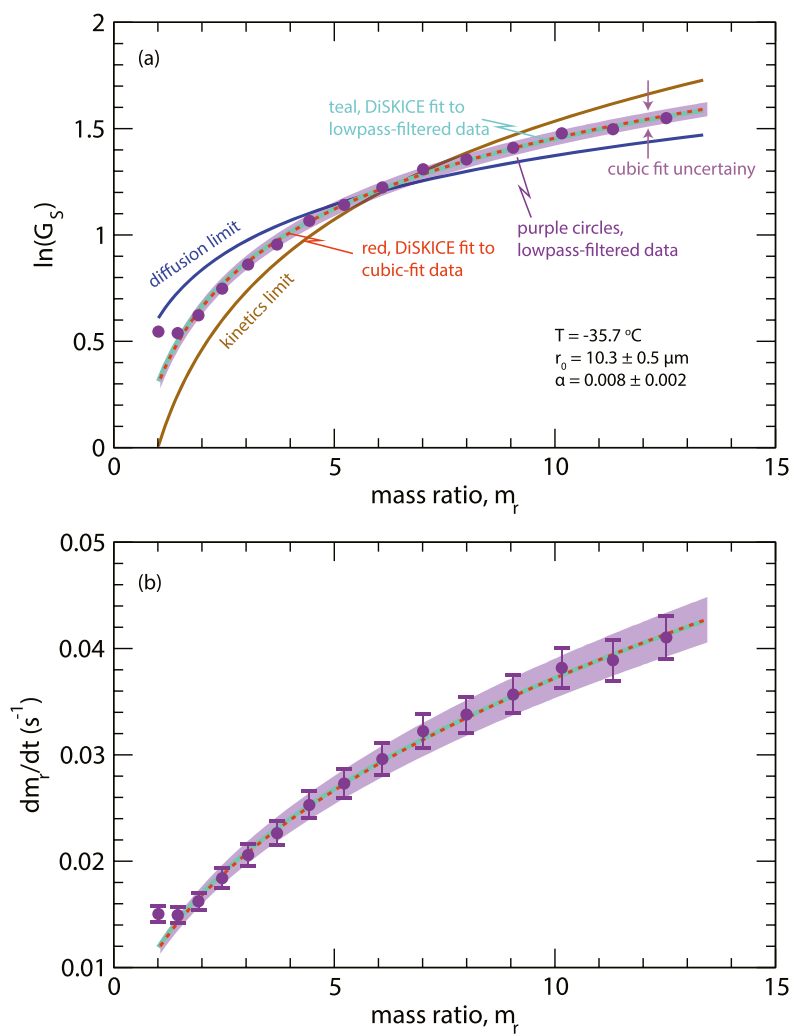

FIG. 7. An example of the scaled mass growth rate analysis with diffusion-kinetics-limited particle growth from heterogeneous freezing data shown in Fig. 6. (a) The natural logarithm of the scaled mass growth rate as a function of the mass ratio. The low-pass-filtered data (purple points) were used to calculate the diffusion (blue) and kinetics (brown) limits. Purple shading is the uncertainty range given by the cubic fit to the data. DiSKICE simulations of the low-pass-filtered (solid teal) and cubic-fit (dashed red) data are shown. (b) Growth rates of the data and model fits shown in (a).

levitated for long periods without changes in size or location. The uptake of foreign gases potentially could inhibit growth, but this effect would most likely diminish, not amplify, as the particle grew. An amplification in the effect would be required to produce a roughly constant growth rate. Though these processes seem unlikely to cause the observed growth rates, there could be other processes occurring of which we are unaware.

At this time, no model exists for the growth of a particle that undergoes a transition from one mode of growth to another. However, the process can be roughly approximated by allowing $M$ [the exponent on $\alpha$ in Eq. (1)] to change as the particle increases in size:

$$
M=1+9\left(\frac{r-r_{0}}{r_{f}-r_{0}}\right)^{3 / 4},
$$
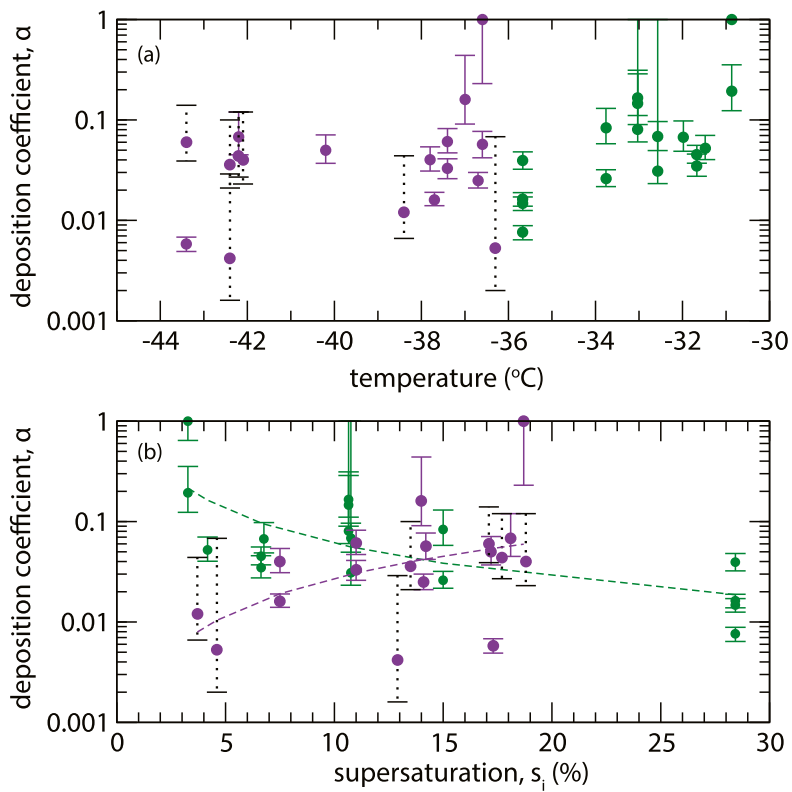

FIG. 8. The deposition coefficient as a function of (a) temperature and (b) supersaturation. Green points are heterogeneous freezing data from Harrison et al. (2016a), and purple points are our homogeneous freezing data. Dotted black error bars indicate the range over which $\alpha$ varies for kinetics transitions with the point located at the mass-ratio weighted mean. The dashed curves in (b) are regression fits to data with the same color.

where $r_{0}$ and $r_{f}$ are the initial and final particle radii, respectively. This model allows particles to begin with efficient growth (spiral dislocations, $M=1$ ) and transition to less-efficient growth (ledge nucleation, $M=10$ ) over time. As a result, $\alpha$ will decline substantially along with the effective diffusivity (dashed curves in Fig. 3). The exponent of $3 / 4$ was chosen to produce a nearly constant mass growth rate, as shown by our measurements. Using this model to fit the data requires the supersaturation-dependent $\alpha$ [Eq. (1)] and, therefore, $s_{\text {char }}$ is determined from the fitting procedure. We emphasize that Eq. (15) is not a physical model of faceting transitions, but instead is a qualitative model that mimics the data and has plausible behavior consistent with a kinetics transition. For example, a spherical crystal may in reality begin with rough growth $(M \sim 0)$ then transition to dislocation growth $(M=1)$. Consequently, the $s_{\text {char }}$ determined with this method should be viewed with caution, since the fitting uses a number of empirical parameters and a hypothetical model [Eq. (15)].

Including Eq. (15) in the DiSKICE model allows us to simulate nearly constant mass growth rates, and fit the growth data shown in Fig. 11. The resulting fits to the scaled growth rates follow the data and reside outside of the diffusion- and kinetics-limit boundaries. The model 

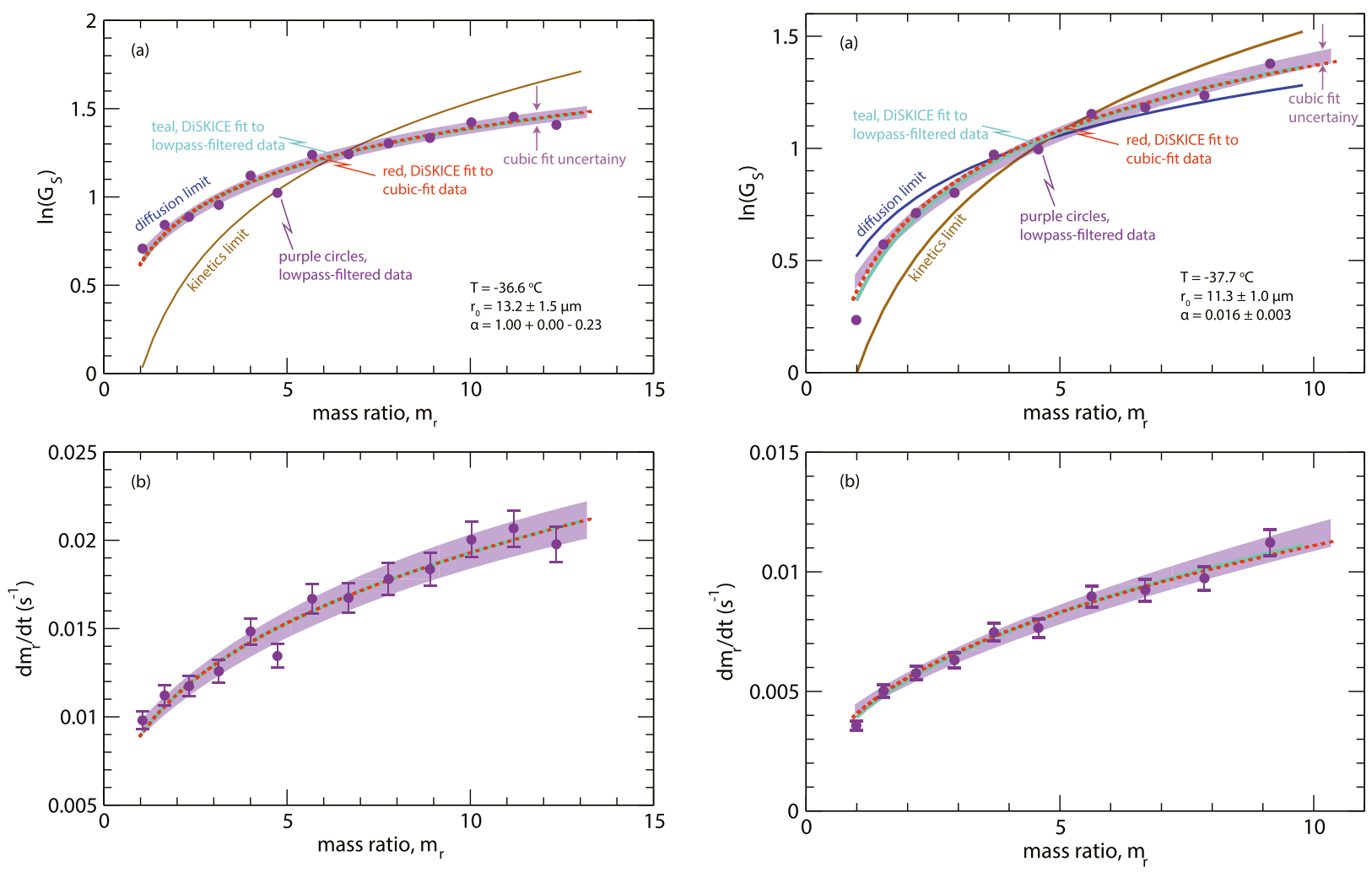

FIG. 9. An example of the scaled mass growth rate analysis with diffusion-limited particle growth from homogeneous freezing data. (a) The natural logarithm of the scaled mass growth rate as a function of the mass ratio. The low-pass-filtered data (purple points) were used to calculate the diffusion (blue) and kinetics (brown) limits. Purple shading is the uncertainty range given by the cubic fit to the data. DiSKICE simulations of the low-pass-filtered (solid teal) and cubic-fit (dashed red) data are shown. (b) Growth rates of the data and model fits shown in (a).

suggests that this is caused by a deposition coefficient that decreases by about an order of magnitude during growth (Fig. 11, $\alpha$ decreases from 0.025 to 0.0016 ). Since $\alpha$ varies greatly during growth for these cases, we show the range of $\alpha$ variability in the error bars. The remaining six experiments, which are all similar to that shown in Fig. 11, were also well fit by this model [Eq. (15)].

The deposition coefficients derived from the homogeneously frozen droplets that can be fit with faceted growth theory [Eqs. (1)-(3)] range from 0.004 to unity, which is similar to the heterogeneously frozen particles, but the experiments that require a kinetics transition [Eq. (15)] cause $\alpha$ to fall below 0.002 by the end of particle growth (Figs. 8 and 11). Furthermore, the rapid decline in $\alpha$ skews the mass-ratio weighted mean $\alpha$ toward the minimum value. All of the $\alpha$ values that we have determined are scattered with temperature (Fig. 8a) as has been shown in prior studies (Skrotzki et al. 2013), but they

FIG. 10. An example of the scaled mass growth rate analysis with diffusion-kinetics-limited particle growth from homogeneous freezing data. (a) The natural logarithm of the scaled mass growth rate as a function of the mass ratio. The low-pass-filtered data (purple points) were used to calculate the diffusion (blue) and kinetics (brown) limits. Purple shading is the uncertainty range given by the cubic fit to the data. DiSKICE simulations of the low-passfiltered (solid teal) and cubic-fit (dashed red) data are shown. (b) Growth rates of the data and model fits shown in (a).

show some supersaturation dependence (Fig. 8b). The deposition coefficients derived from the heterogeneously frozen drops decrease with increasing $s_{i}$, which is counter to the supersaturation dependence expected for faceted growth theory (Fig. 1). However, $\alpha$ values determined from the homogeneous freezing cases increase with $s_{i}$. The potential cause for these differences will be discussed in section 5 .

\section{c. Power-law results}

Qualitatively, the power-law analysis produces similar results to the scaled growth rate analysis, which is noteworthy since the two methods are independent: The scaled growth rate analysis makes no assumption about the functional dependence of $D_{\text {eff }}$, whereas the powerlaw analysis does. All of the heterogeneously frozen drops from Harrison et al. (2016a) and some of our homogeneously frozen drops have $P$ exponents within the range expected for faceted ice growth, as shown for a 

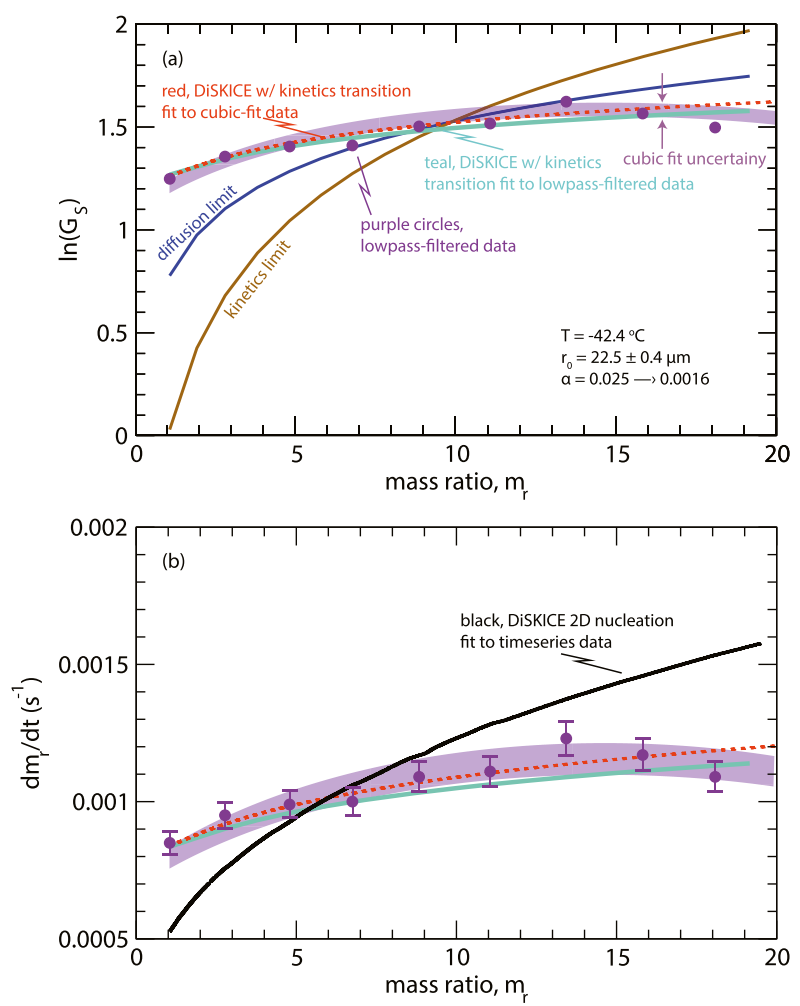

FIG. 11. An example of the scaled mass growth rate analysis with kinetics transitioning particle growth from homogeneous freezing data. (a) The natural logarithm of the scaled mass growth rate as a function of the mass ratio. The low-pass-filtered data (purple points) were used to calculate the diffusion (blue) and kinetics (brown) limits. Purple shading is the uncertainty range given by the cubic fit to the data. DiSKICE simulations using Eq. (15) of the low-pass-filtered (solid teal) and cubic-fit (dashed red) data are shown. The range of $\alpha$ determined from the fit is indicated in the figure. (b) Growth rates of the data and model fits shown in (a). A DiSKICE fit to the mass ratio time series assuming ledge nucleation is given by the black curve.

representative sample in Fig. 12. The curves, which are derived from the data using Eq. (14), are remarkably similar to the theoretical calculations (Fig. 4). Some of these data have $P$ values that begin with values less than 1 , but exceed 1 by the end of growth (green curve in Fig. 12), which may indicate that the particle became nonisometric (see Fig. 4, gray shade). The remaining homogeneous freezing cases have $P<1$ for their entire growth period, similar to the purple curves in Fig. 12, which the faceted growth model cannot reproduce.

For $P<1$, it must be the case that $n<0$ in Eq. (14), which indicates that the effective diffusivity decreases with particle size [see Eq. (12)] as would be expected in a kinetics transition. Indeed, DiSKICE simulations that include a kinetics transition [Eq. (15)] all show a decreasing effective diffusivity with increasing mass (Fig. 3), indicating that the model can reproduce values

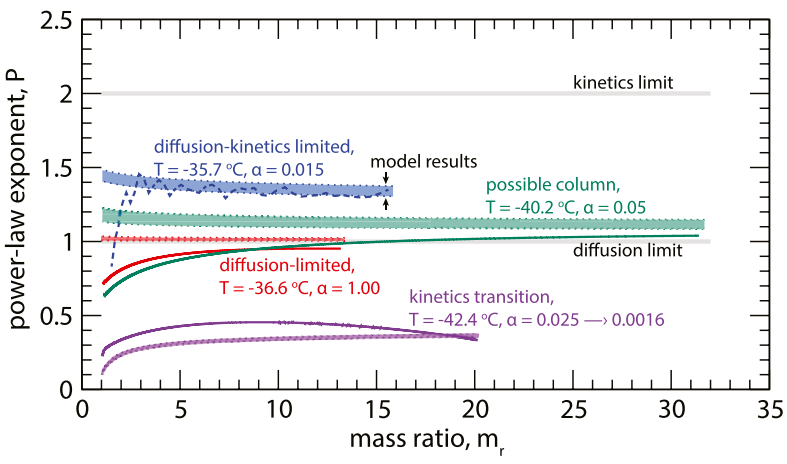

FIG. 12. Power-law exponents as a function of mass ratio as calculated from data. The dashed blue curve is a heterogeneously frozen case using the low-pass-filtered data, and the solid curves are from homogeneously frozen cases with the cubic-fit data. Shaded regions between the dotted lines are calculated from the DiSKICE model fits to the mass ratio uncertainties. Shown here are examples of diffusion-kinetics-limited growth (blue), diffusion-limited growth (red), possible columnar growth (green), and growth with a kinetics transition (purple).

of $P<1$. All seven of the homogeneously frozen crystals that appear to be undergoing kinetics transitions have $P<1$.

We should expect $P$ to be correlated with $\alpha$ if crystals undergo classical faceted growth. Indeed, the $P$ values determined from the data are strongly correlated to the $\alpha$ values derived from the model fits for crystals nucleated heterogeneously, and for homogeneously nucleated crystals that appear to follow faceted growth (Fig. 13). The clear organization of the data is in sharp contrast to the scattered $\alpha-T$ relationship often shown in works on the deposition coefficient (e.g., Skrotzki et al. 2013; and our Fig. 8a). The decline in $P$ with increasing $\alpha$ makes physical sense for faceted growth; since $P=1$ and $\alpha=1$ both pertain to diffusion-limited growth, while $P=2$ and $\alpha \rightarrow 0$ represent kinetics-limited growth. The dependence of $P$ on $\alpha$ is also remarkably consistent with the general shape of the curve derived from Eqs. (1)-(3) for faceted growth (Fig. 13, black curve). This suggests that the model for $\alpha$ is consistent with these growth data. However, the data that indicate the occurrence of kinetics transitions show a different trend, where $P$ seems to increase with $\alpha$, but such a relationship is poorly constrained due to the large temporal variability in both $P$ and $\alpha$ (as indicated by the error bars).

\section{Discussion}

Our results indicate that the method of nucleation may be critical for the early stages of ice vapor-growth. Each time series for heterogeneously frozen ice could be represented by the faceted growth model, but the homogeneously frozen ice requires a kinetics transition 


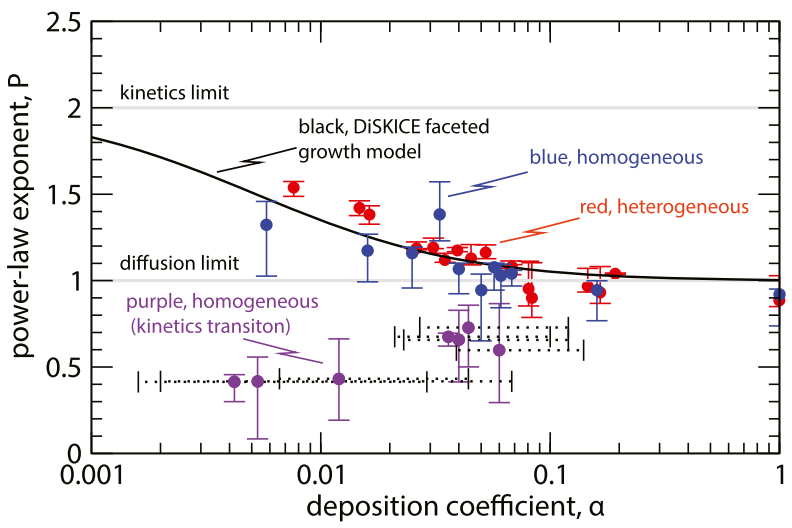

FIG. 13. Power-law exponents as a function of the DiSKICE model-fit deposition coefficient. Points are from the average $P$, with the error bars indicating the maxima and minima throughout growth. Values using heterogeneously (red) and homogeneously frozen ice that follows faceted growth (blue) assume a constant $\alpha$. Data indicative of kinetics transitions (purple) include the ranges over which $\alpha$ varies (dashed black uncertainty) with the points at the mass ratio weighted average values. Plotted over the data are the results of a ledge growth simulation (solid black) with $s_{\text {char }}=$ $10 \%$. The diffusion and kinetics limits are indicated by the gray lines.

model in 7 out of 17 cases. If the HPLC water was somehow contaminated with nuclei in the other 10 cases, causing them to have frozen heterogeneously, then the data would show a distinct divide in the behavior of growth from homogeneously versus heterogeneously frozen drops. While contamination is possible, it seems unlikely given past experiments with this chamber. In past studies, we never succeeded in freezing HPLC water droplets at temperatures above $-36^{\circ} \mathrm{C}$, and this is the main reason Harrison et al. (2016a) used Snomax in their studies.

Despite only a few cases requiring a kinetics transition model, it is likely that all of the measured particles undergo a kinetics transition, since they begin as rough spheres upon which facets emerge. The transitions may simply occur on different time scales. The data that we can simulate with faceted growth may have involved kinetics transitions, but only briefly at the beginning of growth. For instance, it is possible that the heterogeneous ice particles measured by Harrison et al. (2016a) grew into single crystals, which can transition from a sphere to a hexagonal prism within a couple minutes (Gonda and Yamazaki 1984, their Fig. 1). If this occurred, the transition may not be clearly evident in our analysis. In contrast, some of the homogeneously frozen droplets could have formed polycrystals, which is very common at low temperatures. The growth of facets away from the grain boundaries can take tens of minutes (Bacon et al. 2003, their Fig. 8) and, if this occurs, it would be more apparent in our growth data. It is important to note that this assumes that polycrystaline ice would begin with rapid growth. Though intersecting facets can produce rapid growth (Pedersen et al. 2011), it is unknown whether this happens during the emergence of facets (Strickland-Constable 1968, p. 161). Regardless, the kinetics transition for a single crystal may be more challenging to detect, given the shorter transition period, than for a polycrystal. Additionally, the particle's initial size may influence the time scale on which a kinetics transition occurs. For example, one would expect the emergence of facets to require more time over a larger surface area sphere. Indeed, our three largest homogeneously frozen particles $\left(r_{0}=16-27 \mu \mathrm{m}\right)$ all require a kinetics transition model, and most of the remaining homogeneously frozen particles were a few microns larger in initial radius than the heterogeneously frozen particles (cf. in Table 1). Unfortunately, we cannot measure the shape of the small particles, and future work should endeavor to image particle shapes during growth. However, the evidence of kinetics transitions that we have found may help to reconcile the disagreement among prior measurements of the deposition coefficient (e.g., Magee et al. 2006; Skrotzki et al. 2013; Harrison et al. 2016a). Kinetics transitions occurring shortly after freezing would lead to different deposition coefficients being determined depending on if the measurements were made before (high $\alpha$ ), after (low $\alpha$ ), or during (variable $\alpha$ ) the transitions.

Another distinction between the two datasets is how they correlate with supersaturation. The deposition coefficients determined from homogeneously frozen ice rise with the supersaturation, which is as expected from theory (Fig. 1), while the opposite is true for heterogeneous freezing (Fig. 8b). The latter behavior is more clearly shown in the growth rate (Fig. 14), $d m / d t$, when it is normalized by $4 \pi r D$. This normalization reduces the temperature and size dependences, and leaves only the supersaturation and kinetics dependences. To ensure the growth rates normalized by $4 \pi r D$ are robust, we average them over $1-\mu \mathrm{m}$ size ranges $(r=15-16 \mu \mathrm{m}$ in Fig. 14), which makes them independent of the original smoothing method (not shown). Additionally, this normalized growth rate can be compared to theoretical calculations of the maximum diffusion-limited rate, and the faceted growth rate assuming either dislocation or ledge nucleation growth. The heterogeneously frozen particles have increasing normalized growth rates with supersaturation (Fig. 14), though the values are generally below the maximum rate and have a different slope than diffusion-limited theory (Fig. 14, red line). The normalized growth rates at low- $s_{i}$ may be described by dislocation growth (high $\alpha$ ), and are near the maximum 


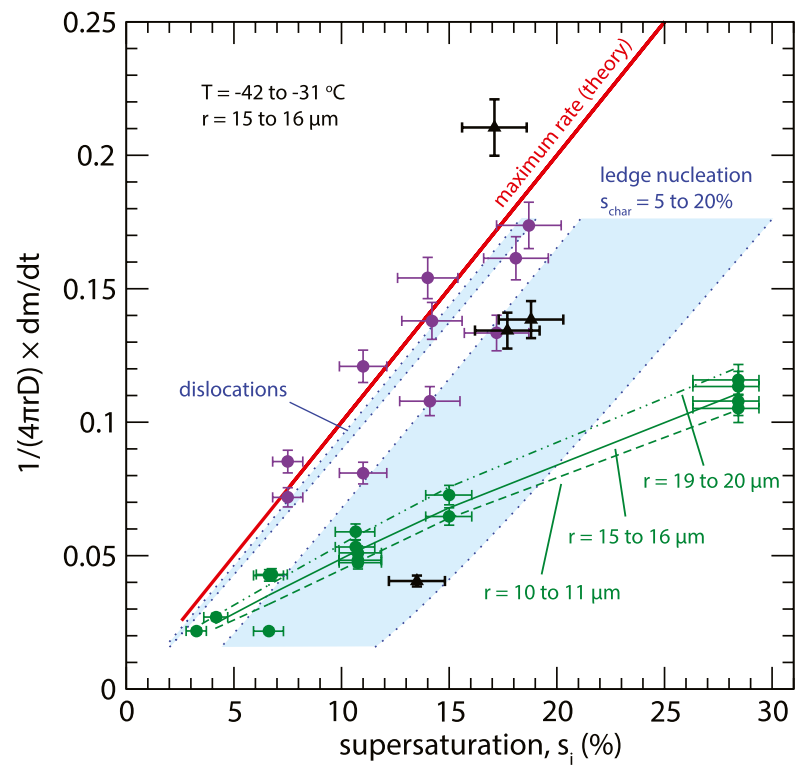

FIG. 14. Effective growth velocity averaged over the period of growth where the particle radius is between 15 and $16 \mu \mathrm{m}$ vs supersaturation. Points are from heterogeneous (green) and homogeneous (purple) freezing data, with black triangles representing particles with kinetics transitions. Green curves are regression fits to the heterogeneous freezing data over the size ranges of 10-11 (dashed), 15-16 (solid), and 19-20 $\mu \mathrm{m}$ (dot-dot-dashed). Theoretical ranges for spherical growth with dislocation and ledge nucleation ( $s_{\text {char }}$ between $5 \%$ and $20 \%$ ) are shaded in blue. The maximum growth rate of a sphere, according to capacitance theory is in red.

rate, but the higher- $s_{i}$ data fall roughly into the ledge nucleation growth (low $\alpha$ ) region. The behavior of declining $\alpha$ and normalized growth rates with $s_{i}$ is inconsistent with faceted growth that assumes a single growth mechanism, but it is broadly consistent with prior studies (Nelson and Knight 1998; Harrington et al. 2019). Those studies show that crystals at low $s_{i}$ appear to grow by dislocations, as originally introduced by Burton et al. (1951). Conversely, at high $s_{i}$ (when $s_{i}>s_{\text {char }}$ ), ledges should nucleate readily, and growth can become instead dominated by ledge nucleation (Nelson and Knight 1998). It is also possible that self-perpetuating stacking faults may influence the growth, as Ming et al. (1988) show this growth mode to produce a minimum growth rate at midrange values of $s_{i}$. Unlike heterogeneous freezing, homogeneously frozen particles show a more complex supersaturation dependence of the normalized growth rate. The crystals that can be modeled with faceted growth (Fig. 14, purple circles) show a general increase in the normalized growth rate with $s_{i}$, similar to the trend in $\alpha$ (Fig. 8). The crystals that require a kinetics transition show no consistent dependence on $s_{i}$ (Fig. 14, black triangles).

Figure 14 also demonstrates that the normalized growth rates for heterogeneously frozen droplets increase with

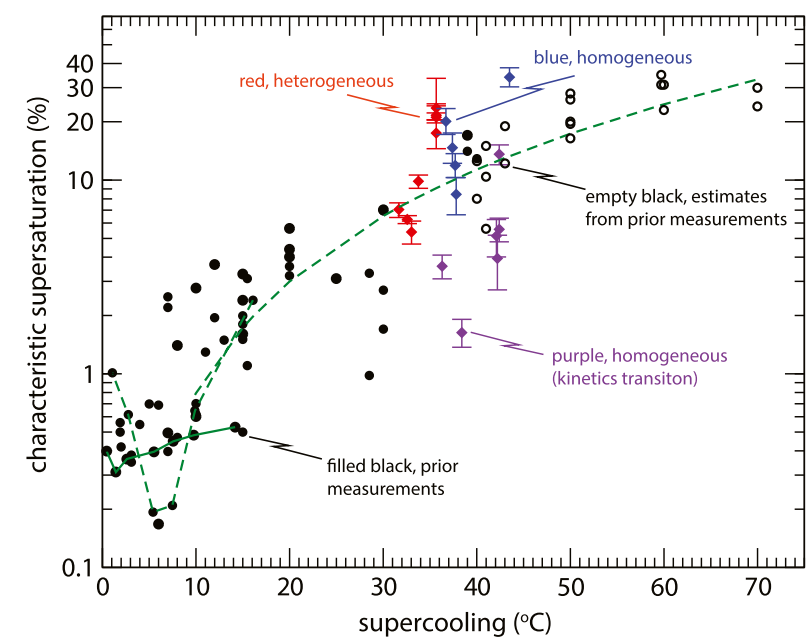

FIG. 15. Characteristic supersaturation vs supercooling (Harrington et al. 2019, based on their Fig. 1). We have added results with influential attachment kinetics $(\alpha<0.05)$ for heterogeneous freezing (red diamonds) and homogeneous freezing with normal faceted growth (blue diamonds) and with kinetics transitions (purple diamonds). Filled black points are from prior faceted growth measurements and empty black circles are estimates from mass growth rates (Harrington et al. 2019).

particle radius as the radius range increases from $10-11$ to $15-16$ to $19-20 \mu \mathrm{m}$, which is not possible with diffusion-limited growth under constant supersaturation [Eq. (2)]. However, this size dependence is consistent with attachment kinetics, since $(1 / r) d m / d t \propto r$ [Eq. (9)] under diffusion-kinetics-limited growth. On the other hand, there is no correlation between the normalized growth rates and size for the homogeneously frozen droplets. Though, some crystals grew near the maximum rate, where a size dependence would be difficult to discern.

We can estimate $s_{\text {char }}$ for some of our data, particularly when $\alpha$ is small $(<0.05)$ and attachment kinetics dominate the growth. This is accomplished by fitting the growth rates with $s_{\text {char }}$ assuming either a ledge growth model $(M=10)$ or a kinetics transition from dislocation to ledge growth $(M \rightarrow 10)$. While our values cover a wide range of $1.63 \%-34.0 \%$ (Fig. 15), the general trend suggests that $s_{\text {char }}$ increases commensurately with supercooling, a result that is consistent with all past studies (Nelson and Knight 1998; Harrington et al. 2019). Interestingly, the data that can be fit using the ledge growth model tend to cluster at higher values of $s_{\text {char }}$ than the data that require kinetics transitions. This is not surprising, since a kinetics transition begins with a reduced kinetic resistance to growth. As with the analysis of kinetics transitions [Eq. (15)], these results depend on the supersaturation, and thus have more substantial uncertainties in comparison to the scaled 
mass growth rate results, and should therefore be treated with caution.

Future studies are required to gain insight into the impacts of ice nucleation and kinetics transitions on the early growth of ice. Most notably, images of growing particles would be useful, even if they are only available at the end of the growth when particles are large. Such images could provide evidence of the type of transition that occurred. Furthermore, growth experiments conducted at lower pressures would be useful: The gasphase resistance for water vapor transport is reduced at low pressure, thus increasing the diffusivity and the particle's sensitivity to the surface kinetics. Under lower pressure, the effects of a kinetics transition should be easier to detect. Finally, ice growth experiments using a variety of ice nucleating particles or solutions could determine if detectable kinetics transitions are truly unique to homogeneously frozen crystals.

\section{Summary}

Theoretical models of ice crystal growth from the vapor are largely unconstrained at temperatures below $-20^{\circ} \mathrm{C}$, due to a lack of sufficient laboratory data. Thus, we measured the mass ratios of homogeneously frozen crystals grown within the button electrode levitation (BEL) diffusion chamber (Harrison et al. 2016a) at temperatures between $-44^{\circ}$ and $-36^{\circ} \mathrm{C}$. These data, and the heterogeneously frozen ice growth data from Harrison et al. (2016a), were analyzed with two new methods that are independent of the ambient supersaturation, a quantity that is challenging to measure in laboratory devices. The first analysis method uses the time average of the mass growth rate instead of the supersaturation. Scaling the growth rate by its mean isolates the effects of the surface attachment kinetics. Fitting the scaled growth rate with the Diffusion Surface Kinetics Ice Crystal Evolution (DiSKICE) model (Zhang and Harrington 2014) then estimates the deposition coefficient, $\alpha$. The second analysis method utilizes the power law dependence in size of the mass growth rate; the power-law exponent can be calculated directly from the data, providing another estimate of the attachment kinetic influences on growth.

We found that the deposition coefficient ranges between 0.002 and unity, with indications of a supersaturation dependence and no dependence on temperature (Fig. 8). Additionally, we found that the method of ice nucleation influenced the growth. Individual time series data from the heterogeneously frozen drops and some of the homogeneously frozen drops could be modeled by faceted growth theory. Modeling the remaining homogeneously frozen drops required a "kinetics transition," in which the growth mechanism changed from efficient (dislocations) to inefficient (ledge nucleation). Prior measurements (e.g., Gonda and Yamazaki 1984; Bacon et al. 2003) show that frozen droplets transform into faceted crystals over time, such that fast-growing regions are replaced with slowgrowing facets, which provides the basis for our modeled kinetics transition. Such a process causes a rapid decline in $\alpha$, rendering the use of a single deposition coefficient value meaningless. All frozen droplets probably experience a kinetics transition as facets emerge, which could in part be responsible for the discrepancy in prior measurements of $\alpha$.

Analysis of mass growth rates normalized by $4 \pi r D$, where $r$ is a $1-\mu \mathrm{m}$-radius range and $D$ is the effective diffusivity showed that the heterogeneously frozen crystals often had normalized growth rates that increased with both size and supersaturation, but were well below the theoretical maximum, which is consistent with kineticslimited growth. On the other hand, the homogeneously frozen crystals demonstrated no size dependence, and the normalized growth rates of those that could be fit with a faceted growth model were often near the theoretical maximum. The homogeneously frozen crystals that required a kinetics transition did not have a consistent supersaturation trend in the normalized growth rate.

Our results suggest that the early growth of ice is significantly impacted by the ice nucleation mechanism, which appears to affect the attachment kinetics. Furthermore, crystals that appear to undergo a kinetics transition have constant growth rates for extended periods of time. Kinetics transitions could influence cloud processes that occur near ice nucleation zones in cold clouds.

Acknowledgments. The authors thank the three anonymous reviewers for their insightful comments that improved this manuscript. The authors are grateful for support from the National Science Foundation under Grant AGS-1824243. Also, the authors benefited from useful conversations with Dr. Dennis Lamb.

Data availability statement: Data for heterogeneously frozen droplet experiments are available at http://www.datacommons.psu.edu/commonswizard/ MetadataDisplay.aspx ?Dataset $=6184$, and data for homogeneously frozen droplet experiments are available at http://www.datacommons.psu.edu/commonswizard/ MetadataDisplay.aspx?Dataset $=6185$.

\section{APPENDIX}

\section{Estimating Supersaturation in the BEL Diffusion Chamber}

Determination of the supersaturation in laboratory devices requires either a direct measurement, or a model that is calibrated with indirect measurements. We 


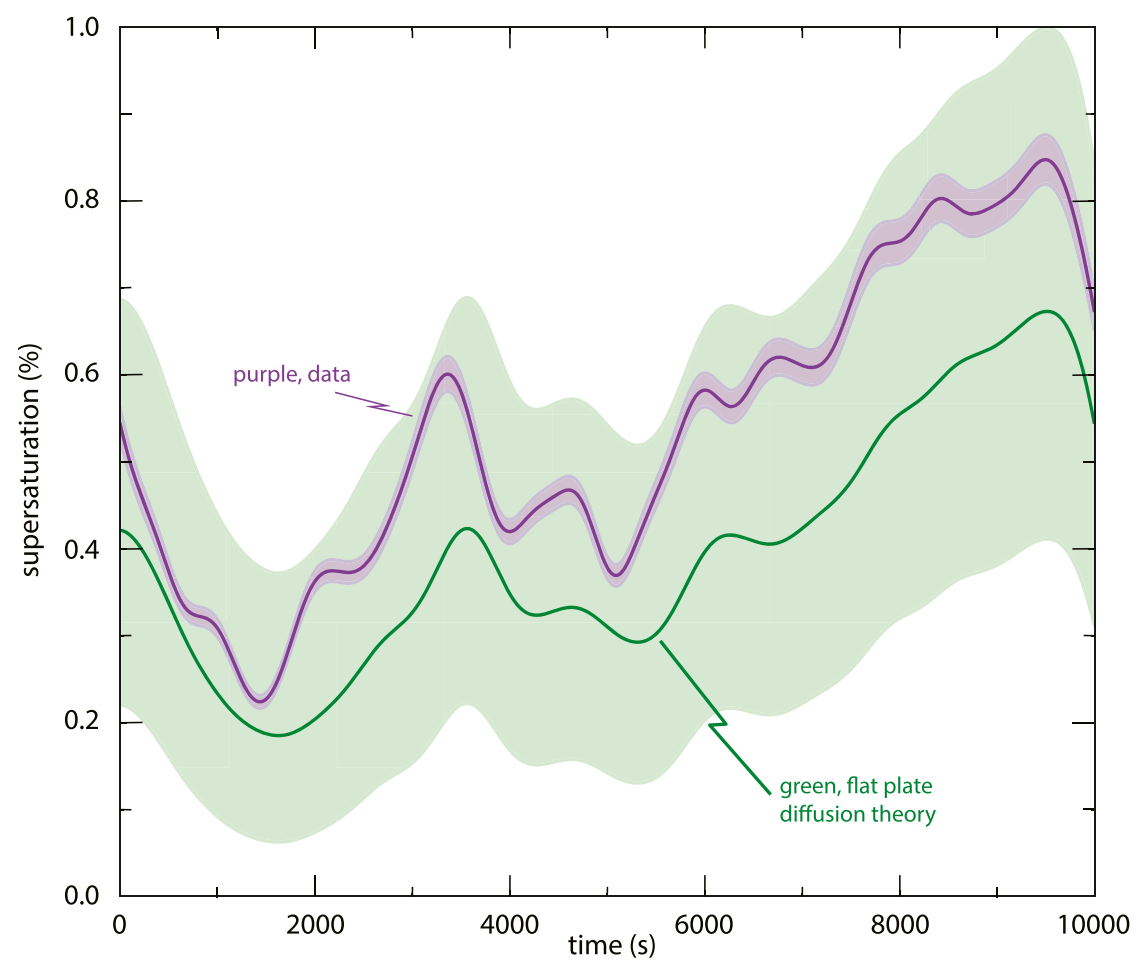

FIG. A1. Supersaturation with a small chamber plate temperature difference $\left(\sim 1^{\circ} \mathrm{C}\right)$. The steady-state flat-plate diffusion chamber theory solution is in green, with $\pm 0.2^{\circ} \mathrm{C}$ error on the measured plate temperatures. The supersaturation estimated from a growing ice particle, assuming that it had a deposition coefficient near unity is in purple. The purple curve assumes that the particle is spherical, with $\pm 0.15 \mu \mathrm{m}$ error on the initial radius.

determine $s_{i}$ in the BEL chamber using a calibrated, two-dimensional diffusion chamber model that assumes that the electrodes and launcher opening areas are not covered with ice (uncovered areas). In Harrison et al. (2016a), this model was calibrated by direct measurements of $s_{i}$ using equilibrated levitated sulfuric acid solution droplets following our prior work (i.e., Xue et al. 2005). In that experiment, $s_{i}$ was determined to be $28.6 \% \pm 1.8 \%$, which is about $12 \%$ lower than the value determined from flat-plate diffusion chamber theory $(32.6 \% \pm 2 \%$; Harrison et al. 2016a, their Fig. 2b). The model was then calibrated by adjusting the uncovered areas until a best fit with the measurements were produced. However, a more recent experiment indicates that this calibration is too aggressive: The calibrated model predicts that the chamber should be subsaturated when the difference in temperatures between the plates is relatively small. This subsaturation remains, though it is slightly smaller, even if a third dimension (horizontal $y$ dimension) is included in the chamber model (not shown). However, as shown below, no such subsaturation is observed with growth measurements under these conditions.

Figure A1 shows a time series of the supersaturation estimated from an experiment conducted with a very small temperature difference $\left(\sim 1^{\circ} \mathrm{C}\right)$ between the plates. This experiment was conducted with a homogeneously frozen HPLC water droplet that grew slowly for nearly $3 \mathrm{~h}$. The particle remained small and so was likely isometric. Moreover, because the crystal was actively growing at very low $s_{i}$ the deposition coefficient must be relatively high, which is consistent with growth theory (see the Introduction). We therefore estimated $s_{i}$ by assuming rough growth $(\alpha=1)$, which should produce a low estimate of $s_{i}$. (The supersaturation would be larger if $\alpha<1$.) The supersaturation determined from the measured growth rate varies from about $0.2 \%$ to $0.8 \%$. For these conditions, the chamber model suggests subsaturated conditions (around $-0.3 \%$ ), which clearly contradicts the growth measurements. We have also included the $s_{i}$ calculated with flat-plate diffusion theory along with the range in that solution produced by including a $\pm 0.2^{\circ} \mathrm{C}$ uncertainty on the plate temperature measurements. Note that the $s_{i}$ estimated from the growth data falls well within the range of the flat-plate solution. This result provides strong evidence that the chamber supersaturation is larger than that determined by the chamber model, and is close to the value determined from flat-plate diffusion theory. While this result 
may seem counterintuitive, it is physically plausible: Gasphase diffusion should cause the opening for the droplet launcher to become nearly saturated with water vapor, and the electrodes may gain an ice coating during experiments.

It is important to note that the upper plate temperature drifted slowly with time in this experiment due a build up of ice in the cryogen housing. However, the temperature changed by less than $0.9^{\circ} \mathrm{C}$ over nearly $3 \mathrm{~h}$. This very slow drift in the temperature produced no transients in the vapor field, since calculations of the supersaturation with the time-dependent diffusion equation are indistinguishable from the steady-state solution (not shown).

To calibrate the chamber model with the experimental result, we reduced the uncovered areas to reproduce the minimum observed $s_{i}$ of $0.21 \% \pm 0.01 \%$. This reduction in area is based on the above argument that the launch opening likely fills in with vapor and that the electrodes may gain a thin layer of frost. When we take into account the uncertainty in the temperature measurement (a maximum plate temperature difference of $1.4^{\circ} \mathrm{C}$ ), we must reduce the uncovered area by $60 \%$ of the physical area, at minimum, to reproduce the measured $s_{i}$. However, reducing the uncovered areas by $60 \%$ produces supersaturations that are not much different from the values determined from flat-plate diffusion theory $(0.13 \%$ vs $0.21 \%$ ). Therefore, it appears that the BEL chamber may be approximated as a flat-plate diffusion chamber.

$\mathrm{Be}$ aware that this result is not inconsistent with the result of Harrison et al. (2016a). Simulations with the chamber model show that the supersaturation at the particle growth location is not very sensitive to the uncovered areas at the high- $s_{i}$ used in their case (about $28 \%$ ). High supersaturations are produced by relatively large plate temperature differences, which tend to drive very rapid diffusion rates. We suspect that this is the reason for the lack of sensitivity to the uncovered area at high $s_{i}$. Hence, it would not be easily possible to detect the effects of the uncovered areas on $s_{i}$ in the experiments of Harrison et al. (2016a). Moreover, as Harrison et al. (2016a) pointed out, using solution drops to calibrate the chamber is potentially problematic in that the ice surface becomes contaminated. Solution contamination on the bottom plate demonstrably lowers $s_{i}$, and could account for the somewhat lower $s_{i}$ measurements of Harrison et al. (2016a) compared to the flat-plate diffusion theory solution.

\section{REFERENCES}

Bacon, N., M. Baker, and B. Swanson, 2003: Initial stages in the morphological evolution of vapour-grown ice crystals: A laboratory investigation. Quart. J. Roy. Meteor. Soc., 129, 1903 1927, https://doi.org/10.1256/qj.02.04.

Beckmann, W., and R. Lacmann, 1982: Interface kinetics of the growth and evaporation of ice single crystals from the vapour phase: II. Measurements in a pure water vapour environment. J. Cryst. Growth, 58, 433-442, https://doi.org/10.1016/00220248(82)90292-5.

Brown, D., S. George, C. Huang, E. Wong, K. Rider, R. Smith, and B. Kay, 1996: $\mathrm{H}_{2} \mathrm{O}$ condensation coefficient and refractive index for vapor-deposited ice from molecular beam and optical interference measurements. J. Phys. Chem., 100, 49884995, https://doi.org/10.1021/jp952547j.

Burton, W. K., N. Cabrera, and F. C. Frank, 1951: The growth of crystals and the equilibrium structure of their surfaces. Philos. Trans. Roy. Soc. London, 243A, 299-358, https://doi.org/ 10.1098/rsta.1951.0006.

Choularton, T., and J. Latham, 1977: Measurements of the deposition coefficient for ice, and its application to cirrus seeding. Quart. J. Roy. Meteor. Soc., 103, 307-318, https://doi.org/ 10.1002/qj.49710343608.

Davis, E., 2010: A button electrode levitation chamber for the study of ice crystal growth under atmospheric conditions. M.S. thesis, Dept. of Meteorology, The Pennsylvania State University, $77 \mathrm{pp}$.

Earle, M., T. Kuhn, A. Khalizov, and J. Sloan, 2010: Volume nucleation rates for homogeneous freezing in supercooled water microdroplets: Results from a combined experimental and modeling appraoch. Atmos. Chem. Phys., 10, 7945-7961, https://doi.org/10.5194/acp-10-7945-2010.

Elliott, W. J., 1971: Dimensions of thermal diffusion chambers. J. Atmos. Sci., 28, 810-811, https://doi.org/10.1175/15200469(1971)028<0810:DOTDC > 2.0.CO;2.

Frank, F. C., 1974: Nucleation-controlled growth on a onedimensional growth of finite length. J. Cryst. Growth, 22, 233-236, https://doi.org/10.1016/0022-0248(74)90100-6.

_ 1982: Snow crystals. Contemp. Phys., 23, 3-22, https://doi.org/ $10.1080 / 00107518208231565$.

Furukawa, Y., and T. Kobayashi, 1978: On the growth mechanism of polycrystalline snow crystals with a specific grain boundary. J. Cryst. Growth, 45, 57-65, https://doi.org/10.1016/00220248(78)90415-3.

Gonda, T., and T. Yamazaki, 1978: Morphology of ice droxtals grown from supercooled water droplets. J. Cryst. Growth, $\mathbf{4 5 ,}$ 66-69, https://doi.org/10.1016/0022-0248(78)90416-5.

$\longrightarrow$, and -1984 : Initial growth forms of snow crystals growing from frozen cloud droplets. J. Meteor. Soc. Japan, 62, 190-192, https://doi.org/10.2151/jmsj1965.62.1_190.

Harrington, J. Y., R. Carver, and D. Lamb, 2009: Parameterization of surface kinetic effects for bulk microphysical models: Influences on simulated cirrus dynamics and structure. J. Geophys. Res., 114, D06212, https://doi.org/10.1029/2008JD011050.

_ - A. Moyle, L. E. Hanson, and H. Morrison, 2019: On calculating deposition coefficients and aspect-ratio evolution in approximate models of ice crystal vapor growth. J. Atmos. Sci., 76, 1609-1625, https://doi.org/10.1175/JAS-D-18-0319.1.

Harrison, A., A. Moyle, M. Hanson, and J. Harrington, 2016a: Levitation diffusion chamber measurements of the mass growth of small ice crystals from vapor. J. Atmos. Sci., 73, 2743-2758, https://doi.org/10.1175/JAS-D-15-0234.1.

$\longrightarrow,-$, and J. Harrington, 2016b: Electrodynamic levitation diffusion chamber measurements of the mass growth of heterogeneously-nucleated ice crystals grown from the vapor. Penn State Data Commons, accessed 1 June 2019, https:// doi.org/10.26208/dd1w-wa17.

Haynes, D., N. Tro, and S. George, 1992: Condensation and evaporation of water on ice surfaces. J. Phys. Chem., 96, 85028509, https://doi.org/10.1021/j100200a055. 
Kong, X., E. Thomson, P. Papagiannakopoulos, S. Johansson, and J. Petterson, 2014: Water accommodation on ice and organic surfaces: Insights from environmental molecular beam experiments. J. Phys. Chem., 118, 13 378-13 386, https://doi.org/ 10.1021/jp5044046.

Lamb, D., and J. Verlinde, 2011: Physics and Chemistry of Clouds. Cambridge University Press, $584 \mathrm{pp}$.

Libbrecht, K., 2003: Growth rates of the principal facets of ice between $-10^{\circ} \mathrm{C}$ and $-40^{\circ}$ C. J. Cryst. Growth, 247, 530-540, https://doi.org/10.1016/S0022-0248(02)01996-6.

, and M. Rickerby, 2013: Measurements of surface attachment kinetics for faceted ice crystal growth. J. Cryst. Growth, 377, 18, https://doi.org/10.1016/j.jcrysgro.2013.04.037.

Magee, N., 2006: A laboratory investigation of vapor-grown ice crystals at low atmospheric temperatures. Ph.D. thesis, The Pennsylvania State University, $234 \mathrm{pp}$.

— A. Moyle, and D. Lamb, 2006: Experimental determination of the deposition coefficient of small cirrus-like crystals near $-50^{\circ}$ C. Geophys. Res. Lett., 33, L17813, https://doi.org/ 10.1029/2006GL026665.

Markov, I., 2003: Crystal Growth for Beginners: Fundamentals of Nucleation, Crystal Growth and Epitaxy. World Scientific, $564 \mathrm{pp}$.

Ming, N.-B., K. Tsukamoto, I. Sunagawa, and A. Chernov, 1988: Stacking faults as self-perpetuating step sources. J. Cryst. Growth, 91, 11-19, https://doi.org/10.1016/0022-0248(88) 90360-0.

Nelson, J., 2001: Growth mechanisms to explain the primary and secondary habits of snow crystals. Philos. Mag., 81A, 23372373, https://doi.org/10.1080/01418610108217152.

_ 2005: Branch growth and sidebranching in snow crystals. Cryst. Growth Des., 5, 1509-1525, https://doi.org/10.1021/ cg049685v.

_ of vapor growth and sublimation of small ice crystals in the atmosphere. J. Geophys. Res., 101, 7033-7047, https://doi.org/ 10.1029/95JD03162.

— layer nucleation. J. Atmos. Sci., 55, 1452-1465, https://doi.org/ 10.1175/1520-0469(1998)055<1452:SCHCEB > 2.0.CO;2.

— ice from lateral-type growth. Atmos. Chem. Phys. Discuss., 2019, 1-51, https://doi.org/10.5194/acp-2019-280.

Neshyba, S., J. Adams, K. Reed, P. M. Rowe, and I. Gladich, 2016: A quasi-liquid mediated continuum model of faceted ice dynamics. J. Geophys. Res. Atmos., 121, 14 035-14055, https://doi.org/10.1002/2016JD025458.

Pedersen, C., A. Mihranyan, and M. Stromme, 2011: Surface transition on ice induced by the formation of a grain boundary. PLOS ONE, 6, e24373, https://doi.org/10.1371/ journal.pone.0024373.

Pokrifka, G., A. Moyle, and J. Harrington, 2018: Electrodynamic levitation diffusion chamber measurements of the mass growth of homogeneously-nucleated ice crystals grown from the vapor. Penn State Data Commons, accessed 1 June 2019, https://doi.org/10.26208/z7bf-nq20.

Pratte, P., H. van den Bergh, and M. Rossi, 2006: The kinetics of $\mathrm{H}_{2} \mathrm{O}$ vapor condensation and evaporation on different types of ice in the range 130-210 K. J. Phys. Chem., 110A, 30423058, https://doi.org/10.1021/jp053974s.

Sei, T., and T. Gonda, 1989: The growth mechanism and the habit change of ice crystals growing from the vapor phase. J. Cryst. Growth, 94, 697-707, https://doi.org/10.1016/0022-0248(89) 90094-8.

Skrotzki, J., and Coauthors, 2013: The accommodation coefficient of water molecules on ice-cirrus cloud studies at the AIDA simulation chamber. Atmos. Chem. Phys., 13, 4451-4466, https://doi.org/10.5194/acp-13-4451-2013.

Strickland-Constable, R. F., 1968: Kinetics and Mechanism of Crystallization from the Fluid Phase and of the Condensation and Evaporation of Liquids. Academic Press, 356 pp.

Swanson, B. D., N. Bacon, E. J. Davis, and M. B. Baker, 1999: Electrodynamic trapping and manipulation of ice crystals. Quart. J. Roy. Meteor. Soc., 125, 1039-1058, https://doi.org/ 10.1002/qj.49712555514.

Westbrook, C. D., R. J. Hogan, and A. J. Illingworth, 2008: The capacitance of pristine ice crystals and aggregate snowflakes. J. Atmos. Sci., 65, 206-219, https://doi.org/10.1175/ 2007JAS2315.1.

Wood, S., M. Baker, and D. Calhoun, 2001: New model for the vapor growth of hexagonal ice crystals in the atmosphere. J. Geophys. Res., 106, 4845-4870, https://doi.org/10.1029/2000JD900338.

Xue, H., A. M. Moyle, N. Magee, J. Harrington, and D. Lamb, 2005: Experimental studies of droplet evaporation kinetics: Validation of models for binary and ternary aqueous solutions. J. Atmos. Sci., 62, 4310-4326, https://doi.org/10.1175/ JAS3623.1.

Zhang, C., and J. Harrington, 2014: Including surface kinetic effects in simple models of ice vapor diffusion. J. Atmos. Sci., 71, 372-390, https://doi.org/10.1175/JAS-D-13-0103.1. 